\title{
INVARIANTS AND ISOMORPHISM THEOREMS FOR ZERO-DIVISOR GRAPHS OF COMMUTATIVE RINGS OF QUOTIENTS
}

\author{
JOHN D. LAGRANGE
}

\begin{abstract}
Given a commutative ring $R$ with $1 \neq 0$, the zero-divisor graph $\Gamma(R)$ of $R$ is the graph whose vertices are the nonzero zero-divisors of $R$, such that distinct vertices are adjacent if and only if their product in $R$ is 0 . It is well known that the zero-divisor graph of any ring is isomorphic to that of its total quotient ring. This result fails for more general rings of quotients. In this paper, conditions are given for determining whether the zero-divisor graph of a ring of quotients of $R$ is isomorphic to that of $R$. Examples involving zero-divisor graphs of rationally $\aleph_{0}$-complete commutative rings are studied extensively. Moreover, several graph invariants are studied and applied in this investigation.
\end{abstract}

1. Introduction. Let $R$ be a commutative ring with $1 \neq 0$, and let $Z(R)$ denote the set of zero-divisors of $R$. The zero-divisor graph $\Gamma(R)$ of $R$ is the simple undirected graph with vertices $V(\Gamma(R))=Z(R) \backslash\{0\}$, such that distinct vertices $v, w \in V(\Gamma(R))$ are adjacent if and only if $v w=0$. The notion of a zero-divisor graph was first introduced by Beck in [3]. While he was mainly interested in colorings, we shall investigate the interplay between ring-theoretic and graph-theoretic properties. This approach began in a paper by Anderson and Livingston [2], and has since continued to evolve.

Let $\Gamma_{1}$ and $\Gamma_{2}$ be simple undirected graphs. Then $\Gamma_{1}$ is isomorphic to $\Gamma_{2}$ if there exists an isomorphism $\varphi: V\left(\Gamma_{1}\right) \rightarrow V\left(\Gamma_{2}\right)$, that is, a bijection $\varphi: V\left(\Gamma_{1}\right) \rightarrow V\left(\Gamma_{2}\right)$ such that $v, w \in V\left(\Gamma_{1}\right)$ are adjacent if and only if $\varphi(v), \varphi(w) \in V\left(\Gamma_{2}\right)$ are adjacent. If $\Gamma_{1}$ is isomorphic to $\Gamma_{2}$, then we will write $\Gamma_{1} \simeq \Gamma_{2}$.

2010 AMS Mathematics subject classification. Primary 13A99, 05C25.

Keywords and phrases. Zero-divisor graph, ring of quotients.

Received by the editors on February 8, 2008, and in revised form on January 20, 2013. 
In [1], it is shown that the zero-divisor graph of any ring is isomorphic to that of its total quotient ring. Related theorems on more general rings of quotients are given in [7] and [9]. While the latter investigations treat rings without nonzero nilpotents, this paper extends results to arbitrary commutative rings. However, rings without nonzero nilpotents shall be considered as well.

A ring $R$ is called reduced if it does not have any nonzero nilpotents. We will say that $R$ is decomposable if $R \cong R_{1} \oplus R_{2}$ for some nonzero rings $R_{1}$ and $R_{2}$. If $R$ is not decomposable, then $R$ is indecomposable. A commutative ring $R$ with $1 \neq 0$ is von Neumann regular if, for each $r \in R$, there exists an $s \in R$ such that $r=r^{2} s$ or, equivalently, $R$ is reduced with Krull dimension zero [6, Theorem 3.1].

Given rings $R \subseteq S$ and a subset $A$ of $S$, define $\operatorname{ann}_{R}(A)=$ $\{r \in R \mid r a=0$ for all $a \in A\}$. If $A=\{a\}$, then we will write $\operatorname{ann}_{R}(A)=\operatorname{ann}_{R}(a)$. An equivalence relation on $R$ is given by declaring elements $r, s \in R$ equivalent if and only if $\operatorname{ann}_{R}(r)=\operatorname{ann}_{R}(s)$. The equivalence class of an element $r \in R$ will be denoted by $[r]_{R}$, that is, $[r]_{R}=\left\{s \in R \mid \operatorname{ann}_{R}(r)=\operatorname{ann}_{R}(s)\right\}$. Suppose that $R$ is von Neumann regular. If $r \in R$, say $r=r^{2} s$, then $e_{r}=r s$ is the unique idempotent that satisfies $[r]_{R}=\left[e_{r}\right]_{R}$ (cf., $[7$, Remark 2.4] or the discussion prior to $[\mathbf{1}$, Theorem 4.1]).

In [6], a ring $R$ is said to satisfy (a.c.) (the annihilator condition) if, given any $r, s \in R$, there exists an $x \in R$ such that $\operatorname{ann}_{R}(r, s)=$ $\operatorname{ann}_{R}(x)$. It follows (by induction) that if $A \subseteq R$ is any finite subset, then there exists an $r \in R$ such that $\operatorname{ann}_{R}(A)=\operatorname{ann}_{R}(r)$. We extend this definition and say that a ring $R$ satisfies $\aleph_{\alpha}$-(g.a.c.) (the $\aleph_{\alpha^{-}}$ generalized annihilator condition) if, given any subset $A \subseteq R$ with $|A|<\aleph_{\alpha}$, there exists an $r \in R$ such that $\operatorname{ann}_{R}(A)=\operatorname{ann}_{R}(r)$. We say that $R$ satisfies (g.a.c.) if it satisfies $\aleph_{\alpha}$-(g.a.c.) for every ordinal $\alpha$. Note that the definition in [6] coincides with our definition of $\aleph_{0^{-}}$ (g.a.c.).

A set $D \subseteq R$ is dense in $R$ if $\operatorname{ann}_{R}(D)=\{0\}$. Let $\mathfrak{d}_{1}$ and $\mathfrak{d}_{2}$ be dense ideals of $R$, and suppose that $f_{i} \in \operatorname{Hom}_{R}\left(\mathfrak{d}_{i}, R\right)(i=1,2)$. Then $\mathfrak{d}_{1} \mathfrak{d}_{2}$ is a dense ideal of $R$, and $\left\{f_{1}+f_{2}, f_{1} \circ f_{2}\right\} \subseteq \operatorname{Hom}_{R}\left(\mathfrak{d}_{1} \mathfrak{d}_{2}, R\right)$. Let $F=\cup_{\mathfrak{d}} \operatorname{Hom}_{R}(\mathfrak{d}, R)$, where the union is taken over all dense ideals of $R$. Then $Q(R)=F / \sim$ is a commutative ring, where $f_{1} \sim f_{2}$ if and only if $\left.f_{1}\right|_{D}=\left.f_{2}\right|_{D}$ for some dense set $D$ of $R$. One checks that $R$ is embedded 
in $Q(R)$ by identifying any element $r \in R$ with the equivalence class containing the homomorphism $(s \mapsto r s) \in \operatorname{Hom}_{R}(R, R)$. In [11], Lambek calls $Q(R)$ the complete ring of quotients of $R$.

A ring extension $R \subseteq S$ is called a ring of quotients of $R$ if $f^{-1} R=\{r \in R \mid f r \in R\}$ is dense in $S$ for all $f \in S$. For example, the total quotient ring $T(R)$ of $R$ is a ring of quotients of $R$. To see this, observe that $s R$ is dense in $T(R)$ whenever $r / s \in T(R)$. Suppose that $S$ is a ring of quotients of $R$. Then the correspondence given by identifying an element $f \in S$ with the equivalence class containing $(r \mapsto f r) \in \operatorname{Hom}_{R}\left(f^{-1} R, R\right)$ is an extension of the mapping $R \rightarrow Q(R)$ described above, and embeds $S$ into $Q(R)$. Therefore, every ring of quotients of $R$ can be regarded as a subring of $Q(R)$. It follows that a dense set in $R$ is dense in every ring of quotients of $R$. Also, $R$ has a unique maximal (with respect to inclusion) ring of quotients, which is isomorphic to $Q(R)$ [11, Proposition 2.3.6]. In this paper, isomorphic rings will not be distinguished. In particular, we shall denote the maximal ring of quotients of $R$ by $Q(R)$. Note that a ring $R$ is reduced if and only if $Q(R)$ is von Neumann regular $[4,1.11]$.

Let $\alpha$ be an ordinal. Given any subsets $D_{1}$ and $D_{2}$ of $R$ such that $\left|D_{i}\right|<\aleph_{\alpha}(i=1,2)$, it follows that $\mid\left\{d_{1} d_{2} \mid d_{1} \in D_{1}\right.$ and $d_{2} \in$ $\left.D_{2}\right\} \mid<\aleph_{\alpha}$. Therefore, the set $Q_{\alpha}(R)=\{f \in Q(R) \mid$ there exists a $D \subseteq f^{-1} R$ such that $\operatorname{ann}_{R}(D)=\{0\}$ and $\left.|D|<\aleph_{\alpha}\right\}$ is a commutative ring. Clearly, $Q_{\alpha}(R)$ is a ring of quotients of $R$. Also, there exists an ordinal $\beta$ such that $Q_{\alpha}(R)=Q(R)$ for all $\alpha \geq \beta$. As in [10], we will say that $R$ is rationally $\aleph_{\alpha}$-complete if $R=Q_{\alpha}(R)$. If $R$ is rationally $\aleph_{\alpha}$-complete, then it is easy to see that $R$ is rationally $\aleph_{\beta}$-complete for all $\beta \leq \alpha$. If $R$ is rationally $\aleph_{\alpha}$-complete for all $\alpha$ (i.e., $R=Q(R)$ ), then we will say that $R$ is rationally complete. In [12], Lucas calls $Q_{0}(R)$ the ring of finite fractions of $R$. In [5], Hager and Martinez refer to $Q_{\alpha}(R)$ as the ring of $\aleph_{\alpha}$-quotients of $R$. Examples and fundamental properties of rational $\aleph_{\alpha}$-completions of commutative rings are given in $[\mathbf{1 0}]$.

Let $R \subseteq S \subseteq T$ be rings. Then $T$ is a ring of quotients of $R$ if and only if $T$ is a ring of quotients of $S$ and $S$ is a ring of quotients of $R[4,1.4]$. It follows that $Q(S)=Q(R)$ whenever $R \subseteq S$ is a ring of quotients. Moreover, given any ordinal $\alpha$, it is easy to check that $f^{-1} R \subseteq f^{-1} S$ for all $f \in Q_{\alpha}(R)$. Therefore, if $S$ is a ring of quotients of $R$, then $Q_{\alpha}(R) \subseteq Q_{\alpha}(S)$ for every ordinal $\alpha$. 
The main focus of this paper is on the relationship between the zerodivisor graphs of $R$ and $Q_{\alpha}(R)$ for a commutative ring $R$. In particular, criteria is sought for determining when these graphs are isomorphic. Using the fact that any ring of quotients of $R$ can be embedded in $Q_{\alpha}(R)$ for some $\alpha$, our results extend to all rings of quotients of $R$.

The ring-theoretic foundation for this study is established in a series of lemmas given in Section 2. Furthermore, these results motivate a ring-theoretic characterization of $\aleph_{\alpha}$-complete Boolean algebras (Theorem 2.4). In [8, Lemma 3.1], a graph-theoretic condition (see Theorem $3.3(4))$ is presented for determining when the relation $\Gamma(R) \simeq \Gamma(Q(R))$ holds for a von Neumann regular ring $R$. However, this condition is meaningful only when certain graph-theoretic assumptions (known to be possessed by zero-divisor graphs of von Neumann regular rings) are met. In particular, this condition cannot be employed in the study of zero-divisor graphs of arbitrary rings. In Section 3, we expose the underlying mechanics of this condition. It turns out that $\aleph_{\alpha}$-(g.a.c.) is an appropriate generalizing criterion (Remark 3.8 (1) and Theorem 3.9). In fact, if $R$ is a von Neumann regular ring, then the key graph-theoretic condition of [8, Lemma 3.1] is possessed by $\Gamma(R)$ if and only if $R$ satisfies (g.a.c.) (Theorem 3.3). In an effort to determine the relation $\Gamma(R) \simeq \Gamma\left(Q_{\alpha}(R)\right)$ based on characteristics of $\Gamma(R)$, we investigate the graph-theoretic implications of the property $\aleph_{\alpha}$-(g.a.c.). Any ring that satisfies $\aleph_{\alpha}$-(g.a.c.) has a weak central vertex $\aleph_{\alpha}$-complete zero-divisor graph. If $R$ is a decomposable ring, then $R$ satisfies $\aleph_{\alpha}$-(g.a.c.) if and only if $\Gamma(R)$ is a weak central vertex $\aleph_{\alpha}$-complete graph (Theorem 3.14 and Corollary 3.15). On the other hand, if $R$ is any reduced ring, then $R$ satisfies $\aleph_{\alpha}$-(g.a.c.) if and only if $\Gamma(R)$ is a central vertex $\aleph_{\alpha}$-complete graph (Theorem 3.1 and Corollary 3.13). We conclude Section 3 with a lemma which provides sufficient conditions for the zero-divisor graphs of direct sums to be isomorphic. In Section 4, the results in Section 3 are applied to examples involving $\Gamma\left(Q_{0}(R)\right)$, where $R$ is a total quotient ring such that $R \subsetneq Q_{0}(R) \subsetneq Q(R)$. In particular, four of the five possible relations between $\Gamma(R), \Gamma\left(Q_{0}(R)\right)$ and $\Gamma(Q(R))$ are shown to exist (Theorem 4.2). Furthermore, examples are constructed to show that $\aleph_{\alpha}$-(g.a.c.) is not a necessary condition for the relation $\Gamma(R) \simeq \Gamma\left(Q_{\alpha}(R)\right)$ to hold (Example 4.15 and Example 4.16). 
2. Rings of quotients and the annihilator conditions. In this section, we study the annihilator ideals of a ring of quotients. In particular, it is shown that the annihilator of an element in a ring of quotients of $R$ is the annihilator of an element in $R$ whenever $R$ satisfies (g.a.c.) (Lemma 2.3). We conclude this section with a theorem which characterizes $\aleph_{\alpha}$-complete Boolean algebras (Theorem 2.4).

In [8], the inclusion $[r]_{R} \subseteq[r]_{Q(R)}$ is justified for a reduced ring by noting that the mapping $\operatorname{ann}_{Q(R)}(J) \mapsto \operatorname{ann}_{R}(J \cap R)(J \subseteq Q(R))$ is a well-defined bijection of $\left\{\operatorname{ann}_{Q(R)}(J) \mid J \subseteq Q(R)\right\}$ onto $\left\{\operatorname{ann}_{R}(J) \mid\right.$ $J \subseteq R\}$ [11, Proposition 2.4.3]. Elementary proofs are given when $R$ is von Neumann regular [8, Proposition 2.7]. The following lemma generalizes this observation with a simpler proof.

Lemma 2.1. Let $R$ be a commutative ring. Suppose that $S$ is a ring of quotients of $R$, and let $f_{1}, f_{2} \in S$. Then $\operatorname{ann}_{R}\left(f_{1}\right)=\operatorname{ann}_{R}\left(f_{2}\right)$ if and only if $\operatorname{ann}_{S}\left(f_{1}\right)=\operatorname{ann}_{S}\left(f_{2}\right)$.

Proof. Clearly, $\operatorname{ann}_{S}\left(f_{1}\right)=\operatorname{ann}_{S}\left(f_{2}\right)$ implies that $\operatorname{ann}_{R}\left(f_{1}\right)=$ $\operatorname{ann}_{R}\left(f_{2}\right)$. Suppose that $\operatorname{ann}_{R}\left(f_{1}\right)=\operatorname{ann}_{R}\left(f_{2}\right)$, and let $g \in \operatorname{ann}_{S}\left(f_{1}\right)$. Then $g\left(g^{-1} R\right) \subseteq \operatorname{ann}_{R}\left(f_{1}\right)=\operatorname{ann}_{R}\left(f_{2}\right)$, and hence $f_{2} g \in \operatorname{ann}_{S}\left(g^{-1} R\right)=$ $\{0\}$. That is, $g \in \operatorname{ann}_{S}\left(f_{2}\right)$. A symmetric argument shows that $\operatorname{ann}_{S}\left(f_{2}\right) \subseteq \operatorname{ann}_{S}\left(f_{1}\right)$, and therefore the desired equality holds.

Lemma 2.2. Let $R$ be a commutative ring. Suppose that $S$ is a ring of quotients of $R$, and let $D$ be a dense set in $R$. If $f \in S$, then

$$
\operatorname{ann}_{R}(f)=\cap_{d \in D} \operatorname{ann}_{R}(f d)=\operatorname{ann}_{R}\left(\cup_{d \in D}\{f d\}\right) .
$$

Proof. To prove the first equality, suppose that $r \in \cap_{d \in D} \operatorname{ann}_{R}(f d)$. Then $r f d=0$ for all $d \in D$. That is, $r f \in \operatorname{ann}_{S}(D)=\{0\}$, where the equality holds since $D$ is dense in every ring of quotients of $R$. Thus, $r \in \operatorname{ann}_{R}(f)$. This shows that $\cap_{d \in D} \operatorname{ann}_{R}(f d) \subseteq \operatorname{ann}_{R}(f)$. The reverse inclusion is obvious, and therefore the equality holds.

The second equality is clear.

Lemma 2.3. Let $R$ and $S$ be commutative rings with $R \subseteq S \subseteq Q_{\alpha}(R)$. Suppose that $R$ satisfies $\aleph_{\alpha}-($ g.a.c.). If $f \in S$, then there exists an $r \in R$ such that $[f]_{S}=[r]_{S}$. 
Proof. The inclusion $S \subseteq Q_{\alpha}(R)$ implies there exists a dense set $D \subseteq f^{-1} R$ such that $|D|<\aleph_{\alpha}$. Since $R$ satisfies $\aleph_{\alpha}$-(g.a.c.), there exists an $r \in R$ such that $\operatorname{ann}_{R}\left(\cup_{d \in D}\{f d\}\right)=\operatorname{ann}_{R}(r)$. But $R \subseteq S \subseteq Q_{\alpha}(R)$ implies that $S$ is a ring of quotients of $R$. Then, by Lemma 2.2, it follows that $\operatorname{ann}_{R}(f)=\operatorname{ann}_{R}(r)$. Therefore, Lemma 2.1 implies that $\operatorname{ann}_{S}(f)=\operatorname{ann}_{S}(r)$, i.e., $[f]_{S}=[r]_{S}$.

Given a commutative ring $R$, let $B(R)=\left\{r \in R \mid r^{2}=r\right\}$, that is, let $B(R)$ denote the set of idempotents of $R$. Then the relation $\leq$, defined by $r \leq s$ if and only if $r s=r$, partially orders $B(R)$ and makes $B(R)$ a Boolean algebra with inf as multiplication in $R$, the largest element as 1 , the smallest element as 0 and complementation defined by $r^{\prime}=1-r$. A Boolean algebra $B$ is called $\aleph_{\alpha}$-complete if inf $A$ exists in $B$ for all $A \subseteq B$ with $|A| \leq \aleph_{\alpha}$. If $B$ is $\aleph_{\alpha}$-complete for every ordinal $\alpha$, then $B$ is called complete. By the de Morgan Laws, it follows that $B$ is $\aleph_{\alpha}$-complete if and only if $\sup A$ exists in $B$ for all $A \subseteq B$ with $|A| \leq \aleph_{\alpha}$ (e.g., see [13, Section 20]).

Suppose that $R$ is von Neumann regular. Let $A \subseteq B(R) \subseteq B(Q(R))$. It is known that $B(Q(R))$ is a complete Boolean algebra [4, Theorem 11.9]. Thus, inf $A \in B(Q(R))$. If $R$ satisfies (g.a.c.), then Lemma 2.3 implies that there exists an element $r \in R$ such that $[r]_{Q(R)}=$ $[\inf A]_{Q(R)}$. But $\inf A$ is idempotent, and thus $\inf A=e_{r} \in R$. Hence, $B(R)$ is complete whenever $R$ satisfies (g.a.c.). The converse is also true. The following theorem generalizes these observations (without the hypothesis " $B(Q(R))$ is complete").

Theorem 2.4. Let $R$ be a von Neumann regular ring. Then $B(R)$ is $\aleph_{\alpha}$-complete if and only if $R$ satisfies $\aleph_{\alpha+1}$-(g.a.c.).

Proof. Suppose that $B(R)$ is $\aleph_{\alpha}$-complete. Let $A \subseteq R$ be such that $|A|<\aleph_{\alpha+1}$. Since $|A| \leq \aleph_{\alpha}$, there exists an $e \in B(R)$ such that $e=\sup \left\{e_{a} \mid a \in A\right\}$. In particular, $e \geq e_{a}$ for all $a \in A$. That is, $e_{a}=e e_{a}$ for all $a \in A$.

Clearly, $\operatorname{ann}_{R}(e) \subseteq \operatorname{ann}_{R}\left(e_{a}\right)=\operatorname{ann}_{R}(a)$ for all $a \in A$. Hence, $\operatorname{ann}_{R}(e) \subseteq \operatorname{ann}_{R}(A)$. To show the reverse inclusion, suppose that $r \in \operatorname{ann}_{R}(A)$. Then $e_{a}\left(1-e_{r}\right)=e_{a}$ for all $a \in A$. That is, $e_{a} \leq 1-e_{r}$ for all $a \in A$. Therefore, $e \leq 1-e_{r}$, i.e., $e\left(1-e_{r}\right)=e$. Then $e e_{r}=0$, 
and therefore $r \in \operatorname{ann}_{R}(e)$. Thus, $\operatorname{ann}_{R}(e)=\operatorname{ann}_{R}(A)$, and it follows that $R$ satisfies $\aleph_{\alpha+1}$-(g.a.c.).

Conversely, suppose that $R$ satisfies $\aleph_{\alpha+1^{-}}$(g.a.c.). Let $A \subseteq B(R)$ be such that $|A| \leq \aleph_{\alpha}$. Since $|A|<\aleph_{\alpha+1}$, there exists an $r \in R$ such that $\operatorname{ann}_{R}(r)=\operatorname{ann}_{R}(\{1-a \mid a \in A\})$. Hence, $\operatorname{ann}_{R}\left(e_{r}\right)=$ $\operatorname{ann}_{R}(\{1-a \mid a \in A\})$. In particular, $\left(1-e_{r}\right)(1-a)=0$ for all $a \in A$. It follows that $1-e_{r} \leq a$ for all $a \in A$. Suppose that $b \in B(R)$ with $b \leq a$ for all $a \in A$. Then $b(1-a)=0$ for all $a \in A$, that is, $b \in \operatorname{ann}_{R}(\{1-a \mid a \in A\})=\operatorname{ann}_{R}\left(e_{r}\right)$. Thus, $b\left(1-e_{r}\right)=b$, i.e., $b \leq 1-e_{r}$. Hence, $\inf A=1-e_{r} \in B(R)$. Therefore, $B(R)$ is $\aleph_{\alpha}$-complete.

Note that Theorem 2.4 gives ring-theoretic conditions which characterize $\aleph_{\alpha}$-complete Boolean algebras. Every Boolean algebra is of the form $B(R)$ for some Boolean ring $R$ (that is, a ring $R$ such that $r^{2}=r$ for all $r \in R$ ), cf., [11, Proposition 1.1.3]. Therefore, a Boolean algebra $B(R)$ is $\aleph_{\alpha}$-complete if and only if $R$ satisfies $\aleph_{\alpha+1}$-(g.a.c.). In Section 3, this ring-theoretic property will be translated into a graphtheoretic property (Theorem 3.1 and Theorem 3.14).

3. Invariants and isomorphism theorems. Let $\Gamma$ be a graph, $V(\Gamma)$ the set of vertices of $\Gamma$ and $\emptyset \neq A \subseteq V(\Gamma)$. As in [8], a vertex $v \in V(\Gamma)$ will be called a central vertex of $A$ if every element of $A$ is adjacent to $v$. Let $C(A) \subseteq V(\Gamma)$ denote the set of all central vertices of $A$. If $A=\{a\}$, then we will write $C(A)=C(a)$. Note that, if $\Gamma=\Gamma(R)$ for some ring $R$, then

$$
C(A)=\operatorname{ann}_{R}(A) \backslash(A \cup\{0\})
$$

A graph $\Gamma$ is said to be central vertex $\aleph_{\alpha}$-complete, or c.v.- $\aleph_{\alpha}$ complete, if, for all $\emptyset \neq A \subseteq V(\Gamma)$ with $|A|<\aleph_{\alpha}$ and $C(A) \neq \emptyset$, there exists a $v \in V(\Gamma)$ such that $C(A)=C(v)$. If $\Gamma$ is c.v.- $\aleph_{\alpha}$-complete for every ordinal $\alpha$, then we will say that $\Gamma$ is c.v.-complete. The following theorem translates this definition into ring-theoretic terms.

Theorem 3.1. Let $R$ be a reduced ring. Then $\Gamma(R)$ is c.v.- $\aleph_{\alpha}$-complete if and only if $R$ satisfies $\aleph_{\alpha}$-(g.a.c.). 
Proof. Observe that, since $R$ is reduced, $C(A)=\operatorname{ann}_{R}(A) \backslash\{0\}$ for every $\emptyset \neq A \subseteq V(\Gamma(R))$. Therefore, the equality $C(A)=C(B)$ holds for nonempty sets $A, B \subseteq V(\Gamma(R))$ if and only if $\operatorname{ann}_{R}(A)=\operatorname{ann}_{R}(B)$.

Suppose that $R$ satisfies $\aleph_{\alpha}$-(g.a.c.). Let $\emptyset \neq A \subseteq V(\Gamma(R))$ with $|A|<\aleph_{\alpha}$ and $C(A) \neq \emptyset$. Then $C(A)=C(r)$, where $r \in R$ is an element such that $\operatorname{ann}_{R}(A)=\operatorname{ann}_{R}(r)$. Hence, $\Gamma(R)$ is a c.v.- $\aleph_{\alpha}$-complete.

Suppose that $\Gamma(R)$ is c.v.- $\aleph_{\alpha}$-complete. Let $\emptyset \neq A \subseteq R$ with $|A|<\aleph_{\alpha}$. If $\operatorname{ann}_{R}(A)=\{0\}$, then $\operatorname{ann}_{R}(A)=\operatorname{ann}_{R}(1)$. Suppose that $\operatorname{ann}_{R}(A) \neq\{0\}$. If $A=\{0\}$. Then $\operatorname{ann}_{R}(A)=\operatorname{ann}_{R}(0)$. Suppose that $A \neq\{0\}$. Then $\operatorname{ann}_{R}(A) \neq\{0\}$ implies that $\emptyset \neq A \backslash\{0\} \subseteq V(\Gamma(R))$ and $C(A \backslash\{0\}) \neq \emptyset$. Therefore, $\operatorname{ann}_{R}(A)=\operatorname{ann}_{R}(A \backslash\{0\})=\operatorname{ann}_{R}(r)$, where $r$ is any element which satisfies $C(A \backslash\{0\})=C(r)$. Thus, $R$ satisfies $\aleph_{\alpha}$-(g.a.c.).

Note that Theorem 3.1 can fail for rings with nonzero nilpotents. For example, the zero-divisor graph of $\mathbf{Z}_{25}$ is the complete graph on four vertices. In particular, $\Gamma\left(\mathbf{Z}_{25}\right)$ is not c.v. $-\aleph_{0}$-complete. However, $\mathbf{Z}_{25}$ satisfies (g.a.c.) since the annihilator of any set in $\mathbf{Z}_{25}$ is either $\{0\}=\operatorname{ann}_{\mathbf{Z}_{25}}(1)$ or $Z(R)=\operatorname{ann}_{\mathbf{Z}_{25}}(5)$.

By Theorem 2.4 and Theorem 3.1, we have

Corollary 3.2. Let $R$ be a von Neumann regular ring. Then $B(R)$ is $\aleph_{\alpha}$-complete if and only if $\Gamma(R)$ is c.v. $\aleph_{\alpha+1}$-complete.

Let $\Gamma$ be a graph, and suppose that $v \in V(\Gamma)$. As in [1], an element $w \in V(\Gamma)$ will be called a complement of $v$ if $w$ is adjacent to $v$, and no element of $V(\Gamma)$ is adjacent to both $v$ and $w$. A graph $\Gamma$ is complemented if every element of $V(\Gamma)$ has a complement. If $\Gamma$ is a simple graph, then $v$ is a complement of $w$ if and only if the edge $v-w$ is not an edge of any triangle in $\Gamma$. It is known that any reduced total quotient ring $R$ is von Neumann regular if and only if $\Gamma(R)$ is complemented $[\mathbf{1}$, Theorem 3.5].

Note that Corollary 3.2 is a generalization of [8, Lemma 3.1], which states the following: If $R$ is a von Neumann regular ring, then $B(R)$ is a complete Boolean algebra if and only if whenever $\emptyset \neq A \subseteq V(\Gamma(R))$ is a family of vertices with $C(A) \neq \emptyset$, there exists a $v \in C(A)$ such that every complement of $v$ is adjacent to every element of $C(A)$. In 
fact, the terminology c.v.-complete was first given in [8], where a zerodivisor graph was said to be c.v.-complete if it satisfied condition (4) of the following theorem.

Theorem 3.3. The following statements are equivalent for a von Neumann regular ring $R$.

(1) For all $\emptyset \neq A \subseteq R$, there exists a $v \in \operatorname{ann}_{R}(A)$ such that $\operatorname{ann}_{R}(A)=\operatorname{ann}_{R}\left(1-e_{v}\right)$.

(2) $R$ satisfies (g.a.c.).

(3) $\Gamma(R)$ is c.v.-complete.

(4) If $\emptyset \neq A \subseteq V(\Gamma(R))$ is a family of vertices with $C(A) \neq \emptyset$, then there exists a $v \in C(A)$ such that every complement of $v$ is adjacent to every element of $C(A)$.

Proof. Observe that (1) implies (2) by definition, (2) implies (3) by Theorem 3.1 and (3) implies (4) by Corollary 3.2 together with [8, Lemma 3.1]. It remains to show that (4) implies (1).

If $\operatorname{ann}_{R}(A)=\{0\}$, then let $v=0$. Suppose that $\operatorname{ann}_{R}(A) \neq\{0\}$. If $A=\{0\}$, then let $v=1$. If $A \neq\{0\}$, then we can regard $A$ as a nonempty subset of $V(\Gamma(R))$ since $\operatorname{ann}_{R}(A)=\operatorname{ann}_{R}(A \backslash\{0\})$. Also, $\operatorname{ann}_{R}(A) \neq\{0\}$ implies that $C(A) \neq \emptyset$, and therefore there exists a $v \in C(A)$ such that every complement of $v$ is adjacent to every element of $C(A)$. But $\operatorname{ann}_{R}(v)=\operatorname{ann}_{R}\left(e_{v}\right)$ implies that $v$ is adjacent to $1-e_{v} \in B(R)$. Moreover, if $r \in R$ with $r v=0=r\left(1-e_{v}\right)$, then $r=r e_{v}=0$. This shows that $1-e_{v}$ is a complement of $v$, and thus $1-e_{v}$ is adjacent to every element of $C(A)$. It follows that $\operatorname{ann}_{R}(A) \subseteq \operatorname{ann}_{R}\left(1-e_{v}\right)$. But if $r \in \operatorname{ann}_{R}\left(1-e_{v}\right)$, then $r=r e_{v} \in \operatorname{ann}_{R}(A)$, where the containment holds since $v \in C(A)$ and $\operatorname{ann}_{R}(v)=\operatorname{ann}_{R}\left(e_{v}\right)$. Thus, $\operatorname{ann}_{R}\left(1-e_{v}\right) \subseteq \operatorname{ann}_{R}(A)$. Hence, $\operatorname{ann}_{R}(A)=\operatorname{ann}_{R}\left(1-e_{v}\right)$.

Suppose that $R$ is a von Neumann regular ring such that $2 \notin Z(R)$ and $|R|<\aleph_{\omega}$. By [8, Theorem 3.3], $\Gamma(R) \simeq \Gamma(Q(R))$ if and only if $\Gamma(R)$ satisfies condition (4) of Theorem 3.3. Then Theorem 3.3 gives several efficient ways of determining whether the zero-divisor graph of a von Neumann regular ring $R$ is isomorphic to that of $Q(R)$. For example, we have: 
Corollary 3.4. Suppose that $R$ is a von Neumann regular ring such that $2 \notin Z(R)$ and $|R|<\aleph_{\omega}$. Then $\Gamma(R) \simeq \Gamma(Q(R))$ if and only if $R$ satisfies (g.a.c.).

Given any $v \in V(\Gamma)$, define $V_{v}(\Gamma)=\{w \in V(\Gamma) \mid C(w)=C(v)\}$. If $\Gamma=\Gamma(R)$ for some ring $R$, then we will write $V_{r}(\Gamma(R))=V_{r}(R)$. Note that the relation $\sim$ on $V(\Gamma)$ defined by $v \sim w$ if and only if $V_{v}(\Gamma)=V_{w}(\Gamma)$ is an equivalence relation. Let $\Gamma^{*}$ be the graph with vertices $\left\{V_{v}(\Gamma) \mid v \in V(\Gamma)\right\}$, such that $V_{v}(\Gamma)$ and $V_{w}(\Gamma)$ are adjacent in $\Gamma^{*}$ if and only if $v$ and $w$ are adjacent in $\Gamma$. The graph $\Gamma^{*}$ was considered in [1], where it was shown that $\Gamma(R)^{*}$ is the zero-divisor graph of a Boolean ring whenever $R$ is von Neumann regular [1, Proposition 4.5]. In [9], the minimal representation of a graph $\Gamma$ was defined as the graph $\Gamma^{*}$, where the vertex $V_{v}(\Gamma)$ was labeled with the cardinal number $\left|V_{v}(\Gamma)\right|$.

If $\Gamma$ is a simple graph, then every edge of $\Gamma^{*}$ represents a complete bipartite graph (see Figure 1). In particular, any zero-divisor graph can be recovered from its minimal representation. Note that, if $R$ is reduced, then $\Gamma(R)^{*}$ is the graph with vertices $\left\{[r]_{R} \mid r \in Z(R) \backslash\{0\}\right\}$, such that $[r]_{R}$ is adjacent to $[s]_{R}$ if and only if $r s=0$. In fact, $[r]_{R}=V_{r}(R)$ for all $r \in Z(R) \backslash\{0\}$.

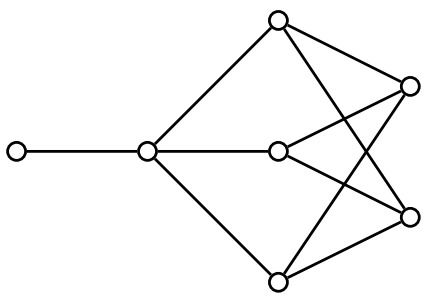

(a) $\Gamma$

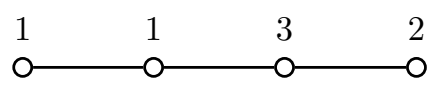

(b) $\Gamma^{*}$

FiguRE 1. A graph $\Gamma$ and its minimal representation $\Gamma^{*}$

Clearly $\Gamma_{1} \simeq \Gamma_{2}$ implies that $\Gamma_{1}^{*} \simeq \Gamma_{2}^{*}$. Although the converse is false, there are certain properties of $\Gamma$ which are preserved by $\Gamma^{*}$. For example, if $n>2$ is an integer, then the diameter of $\Gamma$ is $n$ if and only if 
the diameter of $\Gamma^{*}$ is $n$ (indeed, no two vertices of a minimal path in $\Gamma$ having length $n>2$ can belong to the same vertex in $\left.\Gamma^{*}\right)$. Also, a vertex $w$ is a complement of $v$ in $\Gamma$ if and only if $V_{w}(\Gamma)$ is a complement of $V_{v}(\Gamma)$ in $\Gamma^{*}$. Furthermore, it is a routine exercise to show that a graph $\Gamma$ is c.v.- $\aleph_{\alpha}$-complete if and only if $\Gamma^{*}$ is c.v.- $\aleph_{\alpha}$-complete.

On the other hand, the following proposition gives necessary and sufficient conditions for $\Gamma_{1} \simeq \Gamma_{2}$. Although it was not formally stated, the idea behind Proposition 3.5 was utilized in [1, Theorem 2.2], showing that $\Gamma(R) \simeq \Gamma(T(R))$ for any commutative ring $R$. Moreover, $[\mathbf{1}$, Theorem 4.1] is a special case of this proposition.

Proposition 3.5. Let $\Gamma_{1}$ and $\Gamma_{2}$ be simple undirected graphs. Then $\Gamma_{1} \simeq \Gamma_{2}$ if and only if there exists an isomorphism $\varphi: V\left(\Gamma_{1}^{*}\right) \rightarrow V\left(\Gamma_{2}^{*}\right)$ such that $\left|v^{*}\right|=\left|\varphi\left(v^{*}\right)\right|$ for all $v^{*} \in V\left(\Gamma_{1}^{*}\right)$.

Proof. If $\psi: V\left(\Gamma_{1}\right) \rightarrow V\left(\Gamma_{2}\right)$ is an isomorphism, then it is easy to check that the mapping $\varphi: V\left(\Gamma_{1}^{*}\right) \rightarrow V\left(\Gamma_{2}^{*}\right)$ given by $\varphi\left(V_{v}\left(\Gamma_{1}\right)\right)=$ $V_{\psi(v)}\left(\Gamma_{2}\right)$ has the desired properties. Conversely, suppose that $\varphi$ : $V\left(\Gamma_{1}^{*}\right) \rightarrow V\left(\Gamma_{2}^{*}\right)$ is an isomorphism such that $\left|v^{*}\right|=\left|\varphi\left(v^{*}\right)\right|$ for all $v^{*} \in V\left(\Gamma_{1}^{*}\right)$. For every $v^{*} \in V\left(\Gamma_{1}^{*}\right)$, let $\psi_{v^{*}}: v^{*} \rightarrow \varphi\left(v^{*}\right)$ be a bijection. Then one checks that the mapping $\psi: V\left(\Gamma_{1}\right) \rightarrow V\left(\Gamma_{2}\right)$, given by $\psi(v)=\psi_{v^{*}}(v)$ if and only if $v \in v^{*}$, is an isomorphism.

It is evident from Proposition 3.5 that the cardinality of $V_{r}(R)$ is valuable in determining whether two zero-divisor graphs are isomorphic. If the index of nilpotency of a ring-element $r \in R$ is 2 , then the cardinality of $V_{r}(R)$ is necessarily equal to 1 . This claim is made precise in the following theorem.

Theorem 3.6. Let $R$ be a commutative ring. Suppose that $0 \neq r \in R$ with $r^{2}=0$. Then $V_{r}(R)=\{r\}$.

Proof. Suppose that $x \in V_{r}(R) \backslash\{r\}$. Then $\operatorname{ann}_{R}(x) \backslash\{x\}=$ $\operatorname{ann}_{R}(r) \backslash\{r\}$. In particular, $x r \neq 0$. Thus, $r(1+x) \neq r$. Since $r^{2}=0$, it follows that $r(1+x) \in \operatorname{ann}_{R}(r) \backslash\{r\}=\operatorname{ann}_{R}(x) \backslash\{x\}$. If $1+x$ is not a zero-divisor, then the equality $\operatorname{xr}(1+x)=0$ implies that $x r=0$, a contradiction. Hence, $1+x$ is a zero-divisor. In particular, $x$ is not a nilpotent element. Thus, $\operatorname{ann}_{R}(r) \backslash\{r\}=\operatorname{ann}_{R}(x)$. 
Suppose that $r x \neq r$. Then $r x \in \operatorname{ann}_{R}(r) \backslash\{r\}=\operatorname{ann}_{R}(x)$ implies that $x^{2} \in \operatorname{ann}_{R}(r)$. But $x^{2} \neq r$ since $x$ is not a nilpotent. Thus, $x^{2} \in \operatorname{ann}_{R}(r) \backslash\{r\}=\operatorname{ann}_{R}(x)$, contradicting that $x$ is not a nilpotent. Therefore, it must be the case that $r x=r$.

If $1-x=r$, then $1=x+r=x+r x=x(1+r)$. This contradicts that $x$ is a zero-divisor. Therefore, $1-x \neq r$. Then $r x=r$ implies that $1-x \in \operatorname{ann}_{R}(r) \backslash\{r\}=\operatorname{ann}_{R}(x)$. That is, $x^{2}=x$. Hence, $1+r-x \in \operatorname{ann}_{R}(r)$ and $x(1+r-x)=r \neq 0$. Since $\operatorname{ann}_{R}(r) \backslash\{r\}=\operatorname{ann}_{R}(x)$, it follows that $1+r-x=r$. But then $1-x=0$, and hence $x=1$. This contradicts that $x$ is a zerodivisor, and we have exhausted all possibilities. Therefore, no such element $x$ exists. Thus, $V_{r}(R) \backslash\{r\}=\emptyset$. Clearly, $r \in V_{r}(R)$, and hence $V_{r}(R)=\{r\}$.

Corollary 3.7. Let $R \subseteq S$ be commutative rings. If the mapping $\varphi: V\left(\Gamma(R)^{*}\right) \rightarrow V\left(\Gamma(S)^{*}\right)$ defined by $\varphi\left(V_{r}(R)\right)=V_{r}(S)$ is an isomorphism, then $\left\{r \in R \mid r^{2}=0\right\}=\left\{f \in S \mid f^{2}=0\right\}$.

Proof. Suppose that $0 \neq f \in S$ with $f^{2}=0$. Since $\varphi$ is surjective, there exists an $r \in R$ such that $V_{r}(S)=V_{f}(S)$. But Theorem 3.6 shows that $V_{f}(S)=\{f\}$, and it follows that $f=r \in R$.

For a von Neumann regular ring $R$, condition (4) of Theorem 3.3 is necessary and sufficient to conclude that the mapping $\varphi: V\left(\Gamma(R)^{*}\right) \rightarrow$ $V\left(\Gamma(Q(R))^{*}\right)$ defined by $\varphi\left(V_{r}(R)\right)=V_{r}(Q(R))$ is an isomorphism $([\mathbf{1}$, Proposition 4.5], [4, Theorem 11.9] and [8, Lemma 3.1]). When trying to generalize this result to arbitrary rings, one is forced to seek other criteria. For example, any application of Theorem 3.3 (4) is contingent upon the assumption that elements of $V(\Gamma(R))$ have complements. Remark 3.8(1) and Theorem 3.9 provide generalizations by considering condition (2) of Theorem 3.3.

Remark 3.8. (1) Let $R \subseteq S$ be commutative rings. Suppose that the correspondence $\left\{[r]_{R} \mid 0 \neq r \in Z(R)\right\} \rightarrow\left\{[f]_{S} \mid 0 \neq f \in Z(S)\right\}$ given by $[r]_{R} \mapsto[r]_{S}$ is a bijection, and that $\left|[r]_{R}\right|=\left|[r]_{S}\right|$ for all $0 \neq r \in Z(R)$. Then a proof similar to that of the converse statement in Proposition 3.5 shows that $\Gamma(R) \simeq \Gamma(S)$ (this is precisely the method of proof used in [1, Theorem 2.2]). In particular, suppose that $R \subseteq$ $S \subseteq Q_{\alpha}(R)$ and that $R$ satisfies $\aleph_{\alpha}$-(g.a.c.). Then the correspondence 
$\left\{[r]_{R} \mid 0 \neq r \in Z(R)\right\} \rightarrow\left\{[f]_{S} \mid 0 \neq f \in Z(S)\right\}$ described above is a well-defined bijection by Lemma 2.1 and Lemma 2.3. Therefore, if $\left|[r]_{R}\right|=\left|[r]_{S}\right|$ for all $0 \neq r \in Z(R)$, then $\Gamma(R) \simeq \Gamma(S)$.

(2) Suppose that the mapping $\varphi$ given in Corollary 3.7 is an isomorphism. Using Corollary 3.7, it is easy to see that $[f]_{S} \subseteq R$ for all $0 \neq f \in S$ with $f^{2}=0$. Also, $V_{f}(S)=[f]_{S}$ whenever $0 \neq f \in Z(S)$ with $f^{2} \neq 0$. Therefore, if $\varphi$ is an isomorphism and $\left|V_{r}(R)\right|=\left|V_{r}(S)\right|$ for all $0 \neq r \in Z(R)$, then the correspondence $\left\{[r]_{R} \mid 0 \neq r \in Z(R)\right\} \rightarrow\left\{[f]_{S} \mid 0 \neq f \in Z(S)\right\}$ described above induces an isomorphism from $V(\Gamma(R))$ onto $V(\Gamma(S))$. The converse is false (e.g., by the proof of [1, Theorem 2.2] and Corollary 3.7, the converse fails for the rings $R=\mathbf{Z}_{4}[X]$ and $S=T(R)$ ). In this sense, the isomorphisms induced by $\varphi$ are stronger than the isomorphisms induced by the mapping $\left\{[r]_{R} \mid 0 \neq r \in Z(R)\right\} \rightarrow\left\{[f]_{S} \mid 0 \neq f \in Z(S)\right\}$ described above.

Theorem 3.9. Let $\alpha$ be an ordinal, and suppose that $R$ and $S$ are commutative rings such that $R \subseteq S \subseteq Q_{\alpha}(R)$. Suppose that $R$ satisfies $\aleph_{\alpha}$-(g.a.c.). Then the mapping $\varphi: V\left(\Gamma(R)^{*}\right) \rightarrow V\left(\Gamma(S)^{*}\right)$ defined by $\varphi\left(V_{r}(R)\right)=V_{r}(S)$ is an isomorphism if and only if $\left\{r \in R \mid r^{2}=0\right\}=$ $\left\{f \in S \mid f^{2}=0\right\}$.

Proof. If $\varphi$ is an isomorphism, then the desired equality holds by Corollary 3.7. Conversely, suppose that $\left\{r \in R \mid r^{2}=0\right\}=\{f \in$ $\left.S \mid f^{2}=0\right\}$. To show that $\varphi$ is well defined, suppose that $r, x \in R$ with $V_{r}(R)=V_{x}(R)$. That is, $\operatorname{ann}_{R}(r) \backslash\{r\}=\operatorname{ann}_{R}(x) \backslash\{x\}$. Let $f \in \operatorname{ann}_{S}(r) \backslash\{r\}$. If $f \in R$, then $f \in \operatorname{ann}_{R}(x) \backslash\{x\} \subseteq \operatorname{ann}_{S}(x) \backslash\{x\}$. Therefore, assume that $f \in S \backslash R$.

By Lemma 2.3, there exists an element $t \in R$ such that $\operatorname{ann}_{S}(t)=$ $\operatorname{ann}_{S}(f)$. If $t=r$, then $f^{2}=0$ since $f r=0$ and $\operatorname{ann}_{S}(r)=\operatorname{ann}_{S}(f)$. But this contradicts that $f \in S \backslash R$ since $\left\{r \in R \mid r^{2}=0\right\}=$ $\left\{f \in S \mid f^{2}=0\right\}$. Hence, $t \neq r$. Since $f r=0$, it follows that $t \in \operatorname{ann}_{R}(r)$. Then $t \in \operatorname{ann}_{R}(r) \backslash\{r\}=\operatorname{ann}_{R}(x) \backslash\{x\}$. Thus, $t x=0$, and therefore $f \in \operatorname{ann}_{S}(x)$. Then the containments $f \in S \backslash R$ and $x \in R$ imply that $f \in \operatorname{ann}_{S}(x) \backslash\{x\}$. This shows that $\operatorname{ann}_{S}(r) \backslash\{r\} \subseteq$ $\operatorname{ann}_{S}(x) \backslash\{x\}$. A symmetric argument proves the reverse inclusion, and thus $\operatorname{ann}_{S}(r) \backslash\{r\}=\operatorname{ann}_{S}(x) \backslash\{x\}$. That is, $V_{r}(S)=V_{x}(S)$. Therefore, $\varphi$ is well-defined. 
Clearly, the equality $\operatorname{ann}_{S}(r) \backslash\{r\}=\operatorname{ann}_{S}(x) \backslash\{x\}$ implies that $\operatorname{ann}_{R}(r) \backslash\{r\}=\operatorname{ann}_{R}(x) \backslash\{x\}$. Thus, $\varphi$ is injective. Also, it is straightforward to verify that $\varphi$ preserves and reflects adjacency relations. It only remains to verify that $\varphi$ is surjective.

Let $V_{f}(S) \in V\left(\Gamma(S)^{*}\right)$. By Lemma 2.3, there exists an element $t \in R$ such that $\operatorname{ann}_{S}(t)=\operatorname{ann}_{S}(f)$. If $f^{2}=0$, then $f \in R$. Thus, $V_{f}(S)$ is the image of $V_{f}(R)$. Suppose that $f^{2} \neq 0$. Then $t^{2} \neq 0$. Therefore,

$$
\operatorname{ann}_{S}(t) \backslash\{t\}=\operatorname{ann}_{S}(t)=\operatorname{ann}_{S}(f)=\operatorname{ann}_{S}(f) \backslash\{f\} .
$$

Thus, $V_{f}(S)$ is the image of $V_{t}(R)$. Hence, $\varphi$ is surjective.

Remark 3.10. Note that the proof of the converse statement in Theorem 3.9 does not assume the fact that $V_{r}(R)=\{r\}$ for any $0 \neq r \in Z(R)$ with $r^{2}=0$. Of course, this fact is guaranteed by Theorem 3.6. Therefore, the mapping $\varphi$ given in Theorem 3.9 can be shown to be a well-defined bijection by applying Lemma 2.1 and Lemma 2.3 to elements $V_{r}(R)$ with $r^{2} \neq 0$, and then applying Theorem 3.6 to such elements with $r^{2}=0$.

If $R$ is reduced, then $Q(R)$ satisfies (g.a.c.) by Theorem 2.4 and [4, Theorem 11.9]. However, this observation does not generalize. For example, there exists a reduced ring $R$ such that $Q_{0}(R)$ does not satisfy $\aleph_{0}$-(g.a.c.) (see Example 4.16). Moreover, there exists a ring $R$ containing nonzero nilpotents such that $Q(R)$ does not satisfy $\aleph_{0^{-}}$ (g.a.c.) (see Example 4.15). In particular, the hypothesis $\aleph_{\alpha}$-(g.a.c.) is not a necessary condition for the conclusion of Theorem 3.9.

The following corollary is an immediate consequence of Proposition 3.5, Corollary 3.7 and Theorem 3.9.

Corollary 3.11. Let $\alpha$ be an ordinal, and suppose that $R$ and $S$ are commutative rings such that $R \subseteq S \subseteq Q_{\alpha}(R)$. Suppose that $R$ satisfies $\aleph_{\alpha}$-(g.a.c.) and $\left\{r \in R \mid r^{2}=0\right\}=\left\{f \in S \mid f^{2}=0\right\}$. If $\left|V_{r}(R)\right|=\left|V_{r}(S)\right|$ for all $r \in Z(R)$ with $r^{2} \neq 0$, then $\Gamma(R) \simeq \Gamma(S)$.

If $R$ is reduced, then the hypotheses of Theorem 3.9 are reflected by $\Gamma(R)$. This is made evident in the following corollary. 
Corollary 3.12. Let $\alpha$ be an ordinal, and suppose that $R$ and $S$ are reduced commutative rings such that $R \subseteq S \subseteq Q_{\alpha}(R)$. If $\Gamma(R)$ is c.v.$\aleph_{\alpha}$-complete, then the mapping $\varphi: V\left(\Gamma(R)^{*}\right) \rightarrow V\left(\Gamma(S)^{*}\right)$ defined by $\varphi\left([r]_{R}\right)=[r]_{S}$ is an isomorphism.

Proof. Observe that $\left\{r \in R \mid r^{2}=0\right\}=\emptyset=\left\{f \in S \mid f^{2}=0\right\}$ since $R$ and $S$ are reduced. Moreover, $[r]_{R}=V_{r}(R)$ and $[r]_{S}=V_{r}(S)$ for all $0 \neq r \in Z(R)$. The result now follows from Theorem 3.1 and Theorem 3.9.

Note that Example 4.16 shows that the converse to Corollary 3.12 is false. The following corollary is an immediate consequence of Corollary 3.12 and Proposition 3.5.

Corollary 3.13. Let $\alpha$ be an ordinal, and suppose that $R$ and $S$ are reduced commutative rings such that $R \subseteq S \subseteq Q_{\alpha}(R)$. If $\Gamma(R)$ is c.v. $-\aleph_{\alpha}$-complete and $\left|[r]_{R}\right|=\left|[r]_{S}\right|$ for all $r \in Z(R) \backslash\{0\}$, then $\Gamma(R) \simeq \Gamma(S)$.

Let $\Gamma$ be a graph. We will say that $\Gamma$ is weakly central vertex $\aleph_{\alpha}$ complete, or w.c.v.- $\aleph_{\alpha}$-complete, if for all $\emptyset \neq A \subseteq V(\Gamma)$ with $|A|<\aleph_{\alpha}$, either $C(A)=\emptyset$ or there exists a $v \in V(\Gamma)$ such that

$$
C(v) \backslash A=C(A) \backslash\{v\} .
$$

A graph $\Gamma$ will be called w.c.v.-complete if it is w.c.v.- $\aleph_{\alpha}$-complete for every ordinal $\alpha$. Note that every simple c.v.- $\aleph_{\alpha}$-complete graph is w.c.v.- $\aleph_{\alpha}$-complete. In particular, every c.v.- $\aleph_{\alpha}$-complete zero-divisor graph is w.c.v. $\aleph_{\alpha}$-complete. The converse is false. For example, if $\Gamma$ is a complete graph on at least three vertices, then $\Gamma$ is w.c.v.-complete, but not c.v.-complete.

If $\Gamma=\Gamma(R)$ for some ring $R$, then $\Gamma$ is w.c.v.- $\aleph_{\alpha}$-complete if and only if for all $\emptyset \neq A \subseteq R$ with $|A|<\aleph_{\alpha}$, there exists a $v \in R$ such that

$$
\operatorname{ann}_{R}(v) \backslash(A \cup\{v\})=\operatorname{ann}_{R}(A) \backslash(A \cup\{v\}) .
$$

Therefore, if $R$ satisfies $\aleph_{\alpha}$-(g.a.c.), then $\Gamma(R)$ is w.c.v.- $\aleph_{\alpha}$-complete. The following theorem shows that the converse holds whenever $R$ is decomposable. 
Theorem 3.14. Let $\alpha$ be an ordinal, and suppose that $R$ is a decomposable commutative ring. Let $R_{1}$ and $R_{2}$ be nonzero rings such that $R \cong R_{1} \oplus R_{2}$. Then the following statements are equivalent.

(1) $R_{1}$ and $R_{2}$ satisfy $\aleph_{\alpha}$-(g.a.c.).

(2) $R$ satisfies $\aleph_{\alpha}$-(g.a.c.).

(3) $\Gamma(R)$ is w.c.v. $\aleph_{\alpha}$-complete.

In particular, if $\Gamma(R)$ is a w.c.v. $\aleph_{\alpha}$-complete graph, then every direct summand of $R$ has a w.c.v. $\aleph_{\alpha}$-complete zero-divisor graph.

Proof. Without loss of generality, assume that $R=R_{1} \oplus R_{2}$.

To prove (1) implies (2). Suppose that $R_{1}$ and $R_{2}$ satisfy $\aleph_{\alpha}$-(g.a.c.). Let $\emptyset \neq A \subseteq R$ be such that $|A|<\aleph_{\alpha}$. Note that $\left|\pi_{i}(A)\right|<\aleph_{\alpha}$, where $\pi_{i}$ is the usual projection mapping $(i=1,2)$. Let $r_{i} \in R_{i}$ be an element such that $\operatorname{ann}_{R_{i}}\left(r_{i}\right)=\operatorname{ann}_{R_{i}}\left(\pi_{i}(A)\right)$. It is routine to check that $\operatorname{ann}_{R}\left(\left(r_{1}, r_{2}\right)\right)=\operatorname{ann}_{R}(A)$. Thus, $R$ satisfies $\aleph_{\alpha}$-(g.a.c.).

Note that (2) implies (3) by the above comments.

To show (3) implies (1), suppose that $\Gamma(R)$ is w.c.v. $-\aleph_{\alpha}$-complete. Let $\emptyset \neq A \subseteq R_{1}$ with $|A|<\aleph_{\alpha}$. We need to show that there exists an element $r \in R_{1}$ such that $\operatorname{ann}_{R_{1}}(r)=\operatorname{ann}_{R_{1}}(A)$. Then $R_{2}$ will satisfy $\aleph_{\alpha}$-(g.a.c.) by symmetry.

If $A=\{0\}$, then let $r=0$. Suppose that $A \neq\{0\}$. Then $\operatorname{ann}_{R_{1}}(A)=\operatorname{ann}_{R_{1}}(A \backslash\{0\})$, and hence we can assume that $0 \notin A$.

If $\operatorname{ann}_{R_{1}}(A)=\{0\}$, then let $r=1$. Suppose that $\operatorname{ann}_{R_{1}}(A) \neq\{0\}$. Then $A \times\{1\} \subseteq V\left(\Gamma\left(R_{1} \oplus R_{2}\right)\right)$. Also, $(x, 0) \in C(A \times\{1\})$ for all $0 \neq x \in \operatorname{ann}_{R_{1}}(A)$. Since $\Gamma(R)$ is w.c.v. $-\aleph_{\alpha}$-complete, there exists an element $\left(r_{1}, r_{2}\right) \in R$ such that

$$
C\left(\left(r_{1}, r_{2}\right)\right) \backslash(A \times\{1\})=C(A \times\{1\}) \backslash\left\{\left(r_{1}, r_{2}\right)\right\} .
$$

Suppose that $0 \neq x \in \operatorname{ann}_{R_{1}}(A)$. Then $(x, 0) \in \operatorname{ann}_{R}(A \times\{1\})$. If $(x, 0)=\left(r_{1}, r_{2}\right)$, then $0 \notin A$ implies $(0,1) \in C\left(\left(r_{1}, r_{2}\right)\right) \backslash(A \times\{1\})$. But, clearly $(0,1) \notin C(A \times\{1\})$, contradicting the choice of $\left(r_{1}, r_{2}\right)$. Hence, $(x, 0) \neq\left(r_{1}, r_{2}\right)$, and therefore $(x, 0) \in C(A \times\{1\}) \backslash\left\{\left(r_{1}, r_{2}\right)\right\}$. Thus, $(x, 0) \in C\left(\left(r_{1}, r_{2}\right)\right)$. In particular, $x \in \operatorname{ann}_{R_{1}}\left(r_{1}\right)$. Since $0 \in \operatorname{ann}_{R_{1}}\left(r_{1}\right)$, this shows that $\operatorname{ann}_{R_{1}}(A) \subseteq \operatorname{ann}_{R_{1}}\left(r_{1}\right)$.

If $0 \neq x \in \operatorname{ann}_{R_{1}}\left(r_{1}\right)$, then $(x, 0) \in C\left(\left(r_{1}, r_{2}\right)\right) \backslash(A \times\{1\})$. Thus, $(x, 0) \in C(A \times\{1\})$. Hence, $x \in \operatorname{ann}_{R_{1}}(A)$. Since $0 \in \operatorname{ann}_{R_{1}}(A)$, 
this verifies the inclusion $\operatorname{ann}_{R_{1}}\left(r_{1}\right) \subseteq \operatorname{ann}_{R_{1}}(A)$, and it follows that $\operatorname{ann}_{R_{1}}\left(r_{1}\right)=\operatorname{ann}_{R_{1}}(A)$. Therefore, $R_{1}$ satisfies $\aleph_{\alpha^{-}}$(g.a.c.).

To prove the "in particular" statement, suppose that $\Gamma(R)$ is w.c.v.$\aleph_{\alpha}$-complete. Then the result follows from the above argument since every ring satisfying $\aleph_{\alpha}$-(g.a.c.) has a w.c.v.- $\aleph_{\alpha}$-complete zero-divisor graph.

Note that "decomposable" cannot be omitted from the hypothesis in the previous theorem. Specifically, $\Gamma(R)$ may be w.c.v.- $\aleph_{\alpha^{-}}$

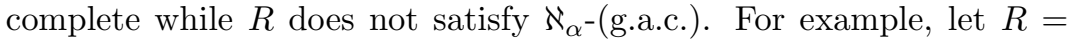
$\mathbf{Z}_{4}[X] /\left(X^{2}\right)$. Then $\Gamma(R)$ is w.c.v.-complete (see Figure 2). Moreover, $\operatorname{ann}_{R}(\{\overline{2}, \overline{2+X}\})=\{\overline{0}, \overline{2 X}\}$. Suppose that $\operatorname{ann}_{R}(f)=\{\overline{0}, \overline{2 X}\}$ for some $f \in R$. Then $f \in\{\overline{0}, \overline{2 X}\}$ since $f^{2}=\overline{0}$ for all $f \in Z(R)$. But then $\overline{2} \in \operatorname{ann}_{R}(f)$, a contradiction. Therefore, no such $f$ exists. Thus, $R$ does not satisfy $\aleph_{0}$-(g.a.c.). Incidentally, we have proved that $R$ is an indecomposable ring. Moreover, any ring having $R$ as a direct summand does not have a w.c.v.- $\aleph_{\alpha}$-complete zero-divisor graph.

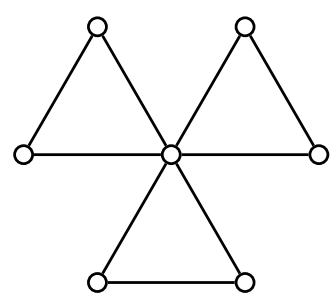

Figure 2. $\Gamma\left(\mathbb{Z}_{4}[X] /\left(X^{2}\right)\right)$

Corollary 3.15. Let $\alpha$ be an ordinal, and suppose that $R$ and $S$ are commutative rings such that $R \subseteq S \subseteq Q_{\alpha}(R)$. Suppose that $R$ is decomposable and $\left\{r \in R \mid r^{2}=0\right\}=\left\{f \in S \mid f^{2}=0\right\}$. If $\Gamma(R)$ is w.c.v.- $\aleph_{\alpha}$-complete and $\left|V_{r}(R)\right|=\left|V_{r}(S)\right|$ for all $r \in Z(R)$ with $r^{2} \neq 0$, then $\Gamma(R) \simeq \Gamma(S)$.

Proof. This result is a restatement of Corollary 3.11, where the $\aleph_{\alpha}$-(g.a.c.) hypothesis has been translated into its graph-theoretic counterpart. 
In Section 4, there are several examples that are constructed by passing to direct sums. We conclude this section with a lemma which will be useful in such constructions.

Lemma 3.16. Let $\varphi_{1}: V\left(\Gamma\left(R_{1}\right)\right) \rightarrow V\left(\Gamma\left(R_{1}^{\prime}\right)\right)$ and $\varphi_{2}: V\left(\Gamma\left(R_{2}\right)\right) \rightarrow$ $V\left(\Gamma\left(R_{2}^{\prime}\right)\right)$ be isomorphisms. If $\left|R_{i} \backslash V\left(\Gamma\left(R_{i}\right)\right)\right|=\left|R_{i}^{\prime} \backslash V\left(\Gamma\left(R_{i}^{\prime}\right)\right)\right|$ for each $i \in\{1,2\}$, then $\Gamma\left(R_{1} \oplus R_{2}\right) \simeq \Gamma\left(R_{1}^{\prime} \oplus R_{2}^{\prime}\right)$.

Proof. Let $\psi_{i}: R_{i} \backslash V\left(\Gamma\left(R_{i}\right)\right) \rightarrow R_{i}^{\prime} \backslash V\left(\Gamma\left(R_{i}^{\prime}\right)\right)$ be bijections with $\psi_{i}\left(0_{R_{i}}\right)=0_{R_{i}^{\prime}}(i=1,2)$. Let $\Phi_{i}: R_{i} \rightarrow R_{i}^{\prime}$ be defined by

$$
\Phi_{i}(r)=\left\{\begin{array}{lc}
\varphi_{i}(r), & r \in V\left(\Gamma\left(R_{i}\right)\right) \\
\psi_{i}(r), & \text { otherwise }
\end{array}\right.
$$

Finally, let $\Psi: R_{1} \oplus R_{2} \rightarrow R_{1}^{\prime} \oplus R_{2}^{\prime}$ be defined by the rule:

$$
\Psi\left(r_{1}, r_{2}\right)=\left(\Phi_{1}\left(r_{1}\right), \Phi_{2}\left(r_{2}\right)\right) .
$$

Then it is straightforward to show that

$$
\left.\Psi\right|_{V\left(\Gamma\left(R_{1} \oplus R_{2}\right)\right)}: V\left(\Gamma\left(R_{1} \oplus R_{2}\right)\right) \longrightarrow V\left(\Gamma\left(R_{1}^{\prime} \oplus R_{2}^{\prime}\right)\right)
$$

is an isomorphism.

4. The zero-divisor graph of $Q_{0}(R)$. Let $R$ be a commutative ring. The zero-divisor graph of $Q(R)$ was studied in [7], where the

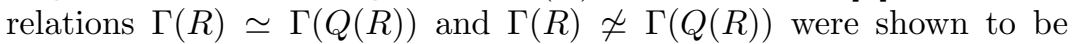
realizable by von Neumann regular rings satisfying $R \subsetneq Q(R)$. With the results of Section 3, we are now equipped to identify relations between more general rings of quotients. In this section, we consider the zero-divisor graphs of $R, Q_{0}(R)$ and $Q(R)$. In particular, we examine the following hypotheses.

Hypothesis 4.1. The following scenarios will be considered for a commutative ring $R$.

(1) $\Gamma(R) \not ॅ \Gamma\left(Q_{0}(R)\right) \not ॅ \Gamma(Q(R))$, and $\Gamma(R) \not \Gamma(Q(R))$.

(2) $\Gamma(R) \simeq \Gamma\left(Q_{0}(R)\right) \not ॅ \Gamma(Q(R))$.

(3) $\Gamma(R) \not \Gamma\left(Q_{0}(R)\right) \simeq \Gamma(Q(R))$.

(4) $\Gamma(R) \simeq \Gamma\left(Q_{0}(R)\right) \simeq \Gamma(Q(R))$.

(5) $\Gamma(R) \simeq \Gamma(Q(R)) \not ॅ \Gamma\left(Q_{0}(R)\right)$. 
Note that the existence of rings which satisfy (2), (3) or (4) of Hypothesis 4.1 can easily be verified. Any $\operatorname{ring} R$ such that $R=Q_{0}(R)$ and $\Gamma(R) \neq \Gamma(Q(R))$ will satisfy relation (2) (e.g., [7, Example 3.7]). Any ring $R$ such that $\Gamma(R) \not \Gamma\left(Q_{0}(R)\right)$ and $Q_{0}(R)=Q(R)$ will satisfy (3) (see Example 4.10). Any rationally complete ring will satisfy (4) (e.g., any finite ring). Furthermore, if $T(R)$ is the total quotient ring of $R$, then $\Gamma(R) \simeq \Gamma(T(R))$ by [1, Theorem 2.2]. Therefore, it is easy to construct examples which satisfy $R \subsetneq T(R)=Q_{0}(R)$ and $\Gamma(R) \simeq \Gamma\left(Q_{0}(R)\right)$ (e.g., let $\left.R=\prod_{\mathbf{N}} \mathbf{Z}\right)$. We shall avoid such trivialities and consider total quotient rings which satisfy $R \subsetneq Q_{0}(R) \subsetneq Q(R)$.

If $\alpha$ is any ordinal, then $Q_{0}\left(Q_{\alpha}(R)\right)=Q_{\alpha}(R)$ by [10, Corollary 3.2]. Therefore, if $T\left(Q_{\alpha}(R)\right)$ is the total quotient ring of $Q_{\alpha}(R)$, then $Q_{\alpha}(R) \subseteq T\left(Q_{\alpha}(R)\right) \subseteq Q_{0}\left(Q_{\alpha}(R)\right)=Q_{\alpha}(R)$. Thus, $T\left(Q_{\alpha}(R)\right)=$ $Q_{\alpha}(R)$. That is, $Q_{\alpha}(R)$ is a total quotient ring for every ordinal $\alpha$.

The results of this section prove the following theorem.

Theorem 4.2. Let $n \in\{1,2,3,4\}$. Then Relation 4.1 (n) can be realized by a total quotient ring $R$ such that $R \subsetneq Q_{0}(R) \subsetneq Q(R)$.

The following examples involve versions of " $A+B$ rings" and "idealizations," as described in Sections 25 and 26 of [6]. All of the graph-isomorphisms of this section are "strong" in the sense of Remark $3.8(2)$. Let $F$ be an infinite field. Set $D_{1}=F[X, Y, Z]$ and $D_{2}=F\left[\left\{X Z^{n}, Y Z^{n} \mid n \geq 0\right\}\right]$, where $X, Y$ and $Z$ are algebraically independent indeterminates. Throughout, $\mathcal{P}$ will be a set of prime ideals of $D_{1}$ containing infinitely many principal ideals. Let $I$ be an indexing set for $\mathcal{P}$. Set $\mathcal{I}=I \times \mathbf{N}$. If $\alpha=(i, n) \in \mathcal{I}$, then set $P_{\alpha}=P_{i}$, and let $K_{\alpha}$ denote the quotient field of $D_{1} / P_{\alpha}$.

Let $\Omega_{k}=\left\{f \in D_{k} \mid f \notin \cup_{\alpha \in \mathcal{I}} P_{\alpha}\right\} \quad(k=1,2)$, and define $\varphi:\left(D_{1}\right)_{\Omega_{1}} \rightarrow \prod_{\alpha \in \mathcal{I}} K_{\alpha}$ to be the canonical homomorphism. Note that $\cap_{\alpha \in \mathcal{I}} P_{\alpha}=\{0\}$ since $D_{1}$ is a unique factorization domain and $\mathcal{P}$ contains infinitely many principal ideals. In particular, $\varphi$ is an embedding. Let $R_{1}=\varphi\left(\left(D_{1}\right)_{\Omega_{1}}\right)+\bigoplus_{\alpha \in \mathcal{I}} K_{\alpha}$ and $R_{2}=\varphi\left(\left(D_{2}\right)_{\Omega_{2}}\right)+\bigoplus_{\alpha \in \mathcal{I}} K_{\alpha}$. Then $R_{2} \subseteq R_{1} \subseteq \prod_{\alpha \in \mathcal{I}} K_{\alpha}$.

Suppose that $\varphi(f / g)+b \in R_{k} \backslash Z\left(R_{k}\right)\left(f \in D_{k}, g \in \Omega_{k}, b \in\right.$ $\left.\bigoplus_{\alpha \in \mathcal{I}} K_{\alpha}, k=1,2\right)$. Then $(\varphi(f / g)+b)(\alpha) \neq \overline{0}$ for all $\alpha \in \mathcal{I}$. Since $b(\alpha)=\overline{0}$ for all but finitely many $\alpha$, it follows that $\varphi(f / g)(\alpha) \neq \overline{0}$ for 
almost all $\alpha$. Thus, $f \notin P$ for all $P \in \mathcal{P}$. That is, $f \in \Omega_{k}$. Hence, $(\varphi(f / g)+b)^{-1}=\varphi(g / f)+b^{\prime} \in R_{k}$, where

$$
b^{\prime}(\alpha)=\left\{\begin{array}{cl}
-\varphi(g / f)(\alpha)+((\varphi(f / g)+b)(\alpha))^{-1}, & b(\alpha) \neq \overline{0} \\
\overline{0}, & \text { otherwise. }
\end{array}\right.
$$

Therefore, $R_{1}$ and $R_{2}$ are total quotient rings. In fact, it will be shown that $R_{1}=Q_{0}\left(R_{1}\right)$ (Proposition 4.7).

Let $J$ be a subset of $\left(D_{k}\right)_{\Omega_{k}}(k=1,2)$. If an element of $R_{k}$ with a nonzero $\alpha$-coordinate annihilates $\varphi(J)$, then $J \subseteq\left(P_{\alpha}\right)_{\Omega_{k}}$. Conversely, if $J \subseteq\left(P_{\alpha}\right)_{\Omega_{k}}$, then the element of $R_{k}$ having a $\overline{1}$ in the $\alpha$-coordinate and $\overline{0}$ elsewhere annihilates $\varphi(J)$. Therefore, $\varphi(J)$ is dense in $R_{k}$ if and only if $J \backslash P_{\Omega_{k}} \neq \emptyset$ for all $P \in \mathcal{P}$.

The dense set $E \subseteq R_{2}$ of elements having a $\overline{1}$ in precisely one coordinate and $\overline{0}$ elsewhere satisfies $E \subseteq r^{-1} R_{2}$ for all $r \in \prod_{\alpha \in \mathcal{I}} K_{\alpha}$. Thus, $\prod_{\alpha \in \mathcal{I}} K_{\alpha} \subseteq Q\left(R_{2}\right)$. As a direct product of fields, $\prod_{\alpha \in \mathcal{I}} K_{\alpha}$ is rationally complete. Hence, $Q\left(R_{2}\right)=\prod_{\alpha \in \mathcal{I}} K_{\alpha}$. Similarly, $Q\left(R_{1}\right)=$ $\prod_{\alpha \in \mathcal{I}} K_{\alpha}$.

The results of this section numbered 4.3-4.7 are derived from proofs found in [6] and [12]. The reader may wish to pass straight to Example 4.8. The following proposition shows that $R_{1}$ satisfies $\aleph_{0}$-(g.a.c.) whenever $\mathcal{P}$ consists entirely of principal ideals (cf., [6, Example 2]).

Proposition 4.3. Let $D$ be a subring of $D_{1}$, and suppose that $\mathcal{P} \subseteq$ $\left\{f D_{1} \mid f \in D\right\}$. Set $\Omega=\{f \in D \mid f \notin P$ for all $P \in \mathcal{P}\}$. Then $\varphi\left(D_{\Omega}\right)+\bigoplus_{\alpha \in \mathcal{I}} K_{\alpha}$ satisfies $\aleph_{0}$-(g.a.c.). In particular, the isomorphism $\Gamma\left(\varphi\left(D_{\Omega}\right)+\bigoplus_{\alpha \in \mathcal{I}} K_{\alpha}\right)^{*} \simeq \Gamma\left(Q_{0}\left(\varphi\left(D_{\Omega}\right)+\bigoplus_{\alpha \in \mathcal{I}} K_{\alpha}\right)\right)^{*}$ holds.

Proof. Let $T=\varphi\left(D_{\Omega}\right)+\bigoplus_{\alpha \in \mathcal{I}} K_{\alpha}$, and suppose that $t_{1}, t_{2} \in T$; say $t_{k}=\varphi\left(f_{k} / g_{k}\right)+b_{k}\left(f_{k} \in D, g_{k} \in \Omega, b_{k} \in \bigoplus_{\alpha \in \mathcal{I}} K_{\alpha}, k=1,2\right)$. Note that the set $\mathcal{I}^{\prime}=\left\{\alpha \in \mathcal{I} \mid\right.$ either $b_{1}(\alpha) \neq \overline{0}$ or $\left.b_{2}(\alpha) \neq \overline{0}\right\}$ is finite. Let $\mathcal{I}^{\prime \prime}=\left\{\alpha \in \mathcal{I}^{\prime} \mid t_{1}(\alpha)=t_{2}(\alpha)=\overline{0}\right\}$. If $f_{1} / g_{1}=f_{2} / g_{2}=0$, then let $f=0$; otherwise, by hypothesis, there exists a (finite) set $J \subseteq D$ such that $\left\{P \in \mathcal{P} \mid\left\{f_{1}, f_{2}\right\} \subseteq P\right\}=\left\{p D_{1} \mid p \in J\right\}$. If $J=\emptyset$, then let $f=1$. Otherwise, let $f=\prod_{p \in J} p \in D$. Define $b \in \prod_{\alpha \in \mathcal{I}} K_{\alpha}$ to be the element 
such that

$$
b(\alpha)= \begin{cases}-\varphi(f)(\alpha), & \alpha \in \mathcal{I}^{\prime \prime} \\ \overline{1}-\varphi(f)(\alpha), & \alpha \in \mathcal{I}^{\prime} \backslash \mathcal{I}^{\prime \prime} \\ \overline{0}, & \text { otherwise }\end{cases}
$$

Note that $b \in \bigoplus_{\alpha \in \mathcal{I}} K_{\alpha}$ since $\mathcal{I}^{\prime}$ is finite. In particular, $\varphi(f)+b \in T$. If $\alpha \in \mathcal{I}^{\prime \prime}$, then $t_{1}(\alpha)=t_{2}(\alpha)=(\varphi(f)+b)(\alpha)=\overline{0}$. If $\alpha \in \mathcal{I}^{\prime} \backslash \mathcal{I}^{\prime \prime}$, then $(\varphi(f)+b)(\alpha)=\overline{1}$, and either $t_{1}(\alpha) \neq \overline{0}$ or $t_{2}(\alpha) \neq \overline{0}$. Suppose that $\alpha \notin \mathcal{I}^{\prime}$. Then $b_{1}(\alpha)=b_{2}(\alpha)=b(\alpha)=\overline{0}$. But, clearly,

$$
\left\{P \in \mathcal{P} \mid\left\{f_{1}, f_{2}\right\} \subseteq P\right\}=\left\{p D_{1} \mid p \in J\right\}=\{P \in \mathcal{P} \mid f \in P\} .
$$

It follows that $\varphi(f)(\alpha)=\overline{0}$ if and only if $\varphi\left(f_{1} / g_{1}\right)(\alpha)=\varphi\left(f_{2} / g_{2}\right)(\alpha)=$ $\overline{0}(\alpha \in \mathcal{I})$. Therefore, $(\varphi(f)+b)(\alpha)=\overline{0}$ if and only if $t_{1}(\alpha)=t_{2}(\alpha)=\overline{0}$. Thus, $\operatorname{ann}_{T}\left(t_{1}, t_{2}\right)=\operatorname{ann}_{T}(\varphi(f)+b)$, and it follows that $T$ satisfies $\aleph_{0^{-}}$ (g.a.c.).

Clearly $T$ is reduced, and hence the "in particular" statement follows from Theorem 3.9.

Proposition 4.4. Suppose that $\mathcal{P}$ is an infinite set of principal prime ideals of $D_{1}$ such that $Z D_{1} \in \mathcal{P}$. Then $R_{2}$ does not satisfy $\aleph_{0}$-(g.a.c.).

Proof. If $R_{2}$ satisfies $\aleph_{0}$-(g.a.c.), then there exists a $t \in R_{2}$ that satisfies the equality $\operatorname{ann}_{R_{2}}(\varphi(X Z), \varphi(Y Z))=\operatorname{ann}_{R_{2}}(t)$. If $f / g \in$ $\left(D_{2}\right)_{\Omega_{2}}$ and $P \in \mathcal{P}$, then $f / g \in P_{\Omega_{2}}$ if and only if $f \in P$. It follows that $t$ can be chosen such that $t=\varphi(f)+b$ for some $f \in D_{2}$ and $b \in \bigoplus_{\alpha \in \mathcal{I}} K_{\alpha}$. Suppose that there exists a $P_{\alpha} \in \mathcal{P}$ such that either $\{X Z, Y Z\} \subseteq P_{\alpha}$ or $f \in P_{\alpha}$, but not both. Say $\alpha=\left(i_{0}, n\right)$. Choose an $N \in \mathbb{N}$ such that $b\left(i_{0}, N\right)=\overline{0}$. Then the element of $R_{2}$ having a $\overline{1}$ in the $\left(i_{0}, N\right)$-coordinate and $\overline{0}$ elsewhere annihilates either $\{\varphi(X Z), \varphi(Y Z)\}$ or $t$, but not both. This is a contradiction. Therefore, $\{X Z, Y Z\} \subseteq P$ if and only if $f \in P(P \in \mathcal{P})$. But $\{X Z, Y Z\} \subseteq P \in \mathcal{P}$ if and only if $P=Z D_{1}$. Thus, $f=u Z^{n}$ for some $0 \neq u \in F$ and $n \geq 1$. This contradicts the containment $f \in D_{2}$. Therefore, no such $f$ exists. Hence, $R_{2}$ does not satisfy $\aleph_{0}$-(g.a.c.).

For any subset $J \subseteq\left(D_{1}\right)_{\Omega_{1}}$, let $J^{-1}$ denote the set of elements in the quotient field of $\left(D_{1}\right)_{\Omega_{1}}$ that map $J$ into $\left(D_{1}\right)_{\Omega_{1}}$ under multiplication. Note that the proofs of Lemma 4.5 and Proposition 4.7 are valid for any set $\mathcal{P}$ of prime ideals of $D_{1}$ which intersect in $\{0\}$. 
Lemma 4.5. Let $J \subseteq\left(D_{1}\right)_{\Omega_{1}}$ be a set such that $J \backslash P_{\Omega_{1}} \neq \emptyset$ for all $P \in \mathcal{P}$. Then $J^{-1}=\left(D_{1}\right)_{\Omega_{1}}$.

Proof. Let $a / b \in J^{-1}$. We can assume that $a, b \in D_{1}$ such that a greatest common divisor of $a$ and $b$ is 1 . Suppose that $a / b \notin\left(D_{1}\right)_{\Omega_{1}}$. Then there exists a $P \in \mathcal{P}$ with $b \in P$. Let $r / q \in J \backslash P_{\Omega_{1}}$. In particular, $r \notin P$. But $(a r) /(b q) \in\left(D_{1}\right)_{\Omega_{1}} \subseteq\left(D_{1}\right)_{P}$ implies that ars $=b q t$ for some $s, t \in D_{1}$ with $s \notin P$. Since $D_{1}$ is a unique factorization domain and $\operatorname{gcd}(a, b)=1$, it follows that $b$ divides $r s$. This contradicts that $r s \notin P$. Therefore, $a / b \in\left(D_{1}\right)_{\Omega_{1}}$. This verifies that $J^{-1} \subseteq\left(D_{1}\right)_{\Omega_{1}}$. The reverse inclusion is obvious.

Lemma 4.6. Suppose that $\mathcal{P}$ is an infinite set of principal prime ideals. Then $Q_{0}\left(R_{1}\right)=Q_{0}\left(R_{2}\right)$.

Proof. There is no principal prime ideal containing both $X$ and $Y$. Also, every element of $D_{1}$ maps the set $\{X, Y\}$ into $D_{2}$ under multiplication. Therefore, $\{\varphi(a X), \varphi(b Y)\}$ is dense in $R_{2}$ for all $a, b \in \Omega_{1}$. Let $s \in R_{1}$; say $s=\varphi(f / g)+b$ for some $f \in D_{1}, g \in \Omega_{1}$, and $b \in \bigoplus_{\alpha \in \mathcal{I}} K_{\alpha}$. Then $\{\varphi(g X), \varphi(g Y)\} \subseteq \varphi(f / g)^{-1} R_{2}$. Clearly, $\{\varphi(g X), \varphi(g Y)\} \subseteq$ $b^{-1} R_{2}$ (indeed, $b \in R_{2}$ ), and thus $\{\varphi(g X), \varphi(g Y)\} \subseteq s^{-1} R_{2}$. Hence, $s \in Q_{0}\left(R_{2}\right)$. This shows that $R_{1} \subseteq Q_{0}\left(R_{2}\right)$. Moreover, the inclusions $R_{2} \subseteq R_{1} \subseteq Q_{0}\left(R_{2}\right) \subseteq Q\left(R_{2}\right)$ imply that

$$
Q_{0}\left(R_{2}\right) \subseteq Q_{0}\left(R_{1}\right) \subseteq Q_{0}\left(Q_{0}\left(R_{2}\right)\right)=Q_{0}\left(R_{2}\right),
$$

where the equality holds by $\left[\mathbf{1 0}\right.$, Corollary 3.2]. Therefore, $Q_{0}\left(R_{1}\right)=$ $Q_{0}\left(R_{2}\right)$.

The ring $Q_{0}(R)$ is calculated in [12, Theorem 11], where $R$ is a ring constructed using the principle of idealization. The proof of the following proposition is a close mimicry of the one given for $[\mathbf{1 2}$, Theorem 11(d)].

Proposition 4.7. Let $R_{1}$ and $R_{2}$ be defined as above. Then $Q_{0}\left(R_{1}\right)=$ $R_{1}$. If $\mathcal{P}$ consists entirely of principal ideals, then $Q_{0}\left(R_{2}\right)=R_{1}$.

Proof. By Lemma 4.6, it suffices to show that $Q_{0}\left(R_{1}\right)=R_{1}$. Suppose that $s \in Q_{0}\left(R_{1}\right)$. There exists a finite set $J=\left\{j_{1}, \ldots, j_{n}\right\} \subseteq$ 
$\left(D_{1}\right)_{\Omega_{1}} \backslash\{0\}$, and elements $b_{k} \in \bigoplus_{\alpha \in \mathcal{I}} K_{\alpha}(k=1, \ldots, n)$, such that the set

$$
\left\{\varphi\left(j_{1}\right)+b_{1}, \ldots, \varphi\left(j_{n}\right)+b_{n}\right\}
$$

is dense and contained in $s^{-1} R_{1}$. It follows that $\varphi(J)$ is dense. If not, then $J \subseteq\left(P_{\beta}\right)_{\Omega_{1}}$ for some $\beta=\left(i_{0}, m\right) \in \mathcal{I}$. But the set $\left\{\alpha \in \mathcal{I} \mid b_{k}(\alpha) \neq \overline{0}\right.$ for some $\left.k \in\{1, \ldots, n\}\right\}$ is finite. Hence, there exists an integer $N$ such that $b_{k}\left(\left(i_{0}, N\right)\right)=\overline{0}$ for all $k \in\{1, \ldots, n\}$. Then the nonzero element of $R_{1}$ having a $\overline{1}$ in the $\left(i_{0}, N\right)$-coordinate and $\overline{0}$ elsewhere annihilates $\left\{\varphi\left(j_{1}\right)+b_{1}, \ldots, \varphi\left(j_{n}\right)+b_{n}\right\}$, a contradiction. Therefore, $J \backslash P_{\Omega_{1}} \neq \emptyset$ for all $P \in \mathcal{P}$. Thus, $\varphi(J)$ is dense.

Clearly, $s b_{k} \in \bigoplus_{\alpha \in \mathcal{I}} K_{\alpha} \subseteq R_{1}$ for each $k \in\{1, \ldots, n\}$. Hence, $s \varphi\left(j_{k}\right)=s\left(\varphi\left(j_{k}\right)+b_{k}\right)-s b_{k} \in R_{1}$ for all $k \in\{1, \ldots, n\}$; say

$$
s \varphi\left(j_{k}\right)=\varphi\left(f_{k} / g_{k}\right)+e_{k}
$$

for some $f_{k} \in D_{1}, g_{k} \in \Omega_{1}$, and $e_{k} \in \bigoplus_{\alpha \in \mathcal{I}} K_{\alpha}(k=1, \ldots, n)$. by

Consider the mapping $\psi: \sum_{k=1}^{n} \varphi\left(j_{k}\left(D_{1}\right)_{\Omega_{1}}\right) \rightarrow \varphi\left(\left(D_{1}\right)_{\Omega_{1}}\right)$ defined

$$
\psi\left(\sum_{k=1}^{n} \varphi\left(j_{k} r_{k} / q_{k}\right)\right)=\sum_{k=1}^{n} \varphi\left(\left(f_{k} / g_{k}\right)\left(r_{k} / q_{k}\right)\right), r_{k} \in D_{1}, q_{k} \in \Omega_{1} .
$$

Note that $\psi$ is well-defined since $\sum_{k=1}^{n} \varphi\left(j_{k} r_{k} / q_{k}\right)=(\overline{0})$ implies

$$
\sum_{k=1}^{n} \varphi\left(\left(f_{k} / g_{k}\right)\left(r_{k} / q_{k}\right)\right)+\sum_{k=1}^{n} e_{k} \varphi\left(r_{k} / q_{k}\right)=s \sum_{k=1}^{n} \varphi\left(j_{k} r_{k} / q_{k}\right)=(\overline{0}),
$$

and thus

$$
\sum_{k=1}^{n} \varphi\left(\left(f_{k} / g_{k}\right)\left(r_{k} / q_{k}\right)\right) \in \varphi\left(\left(D_{1}\right)_{\Omega_{1}}\right) \cap \bigoplus_{\alpha \in \mathcal{I}} K_{\alpha}=(\overline{0}) .
$$

Hence $\psi((\overline{0}))=(\overline{0})$. Then, clearly,

$$
\psi \in \operatorname{Hom}_{\varphi\left(\left(D_{1}\right)_{\Omega_{1}}\right)}\left(\sum_{k=1}^{n} \varphi\left(j_{k}\left(D_{1}\right)_{\Omega_{1}}\right), \varphi\left(\left(D_{1}\right)_{\Omega_{1}}\right)\right) .
$$

Choose an element $j \in J$. Then $s_{1}=\psi(\varphi(j)) / \varphi(j)$ belongs to the quotient field of $\varphi\left(\left(D_{1}\right)_{\Omega_{1}}\right)$, and

$$
s_{1} \varphi\left(j_{k}\right)=\psi\left(\varphi\left(j_{k}\right)\right)=\varphi\left(f_{k} / g_{k}\right) \in \varphi\left(\left(D_{1}\right)_{\Omega_{1}}\right)
$$


for all $k \in\{1, \ldots, n\}$. Also, $J^{-1}=\left(D_{1}\right)_{\Omega_{1}}$ by Lemma 4.5, and it follows that $s_{1} \in \varphi\left(\left(D_{1}\right)_{\Omega_{1}}\right)$. by

Consider the mapping $\rho: \sum_{k=1}^{n} \varphi\left(j_{k}\left(D_{1}\right)_{\Omega_{1}}\right) \rightarrow \bigoplus_{\alpha \in \mathcal{I}} K_{\alpha}$ defined

$$
\rho\left(\sum_{k=1}^{n} \varphi\left(j_{k} r_{k} / q_{k}\right)\right)=\sum_{k=1}^{n} e_{k} \varphi\left(r_{k} / q_{k}\right), r_{k} \in D_{1}, q_{k} \in \Omega_{1} .
$$

Note that $\rho$ is well defined since the above computations show that the equality $\sum_{k=1}^{n} e_{k} \varphi\left(r_{k} / q_{k}\right)=(\overline{0})$ holds whenever $\sum_{k=1}^{n} \varphi\left(j_{k} r_{k} / q_{k}\right)=$ $(\overline{0})$. Hence,

$$
\rho \in \operatorname{Hom}_{\varphi\left(\left(D_{1}\right)_{\Omega_{1}}\right)}\left(\sum_{k=1}^{n} \varphi\left(j_{k}\left(D_{1}\right)_{\Omega_{1}}\right), \bigoplus_{\alpha \in \mathcal{I}} K_{\alpha}\right) .
$$

For each $\alpha \in \mathcal{I}$, choose an element $t_{\alpha} \in \varphi\left(J \backslash\left(P_{\alpha}\right)_{\Omega_{1}}\right)$. Let $j_{k} \in J$. Then

$$
t_{\alpha}(\alpha)\left(\rho\left(\varphi\left(j_{k}\right)\right)(\alpha)\right)=\left(\varphi\left(j_{k}\right)(\alpha)\right)\left(\rho\left(t_{\alpha}\right)(\alpha)\right) .
$$

This shows that $\rho\left(\varphi\left(j_{k}\right)\right)=s_{2} \varphi\left(j_{k}\right)$ for all $j_{k} \in J$, where $s_{2} \in \prod_{\alpha \in \mathcal{I}} K_{\alpha}$ is the element such that $s_{2}(\alpha)=t_{\alpha}(\alpha)^{-1}\left(\rho\left(t_{\alpha}\right)(\alpha)\right)$ for all $\alpha \in \mathcal{I}$. That is,

$$
s_{2} \varphi\left(j_{k}\right)=e_{k} \in \bigoplus_{\alpha \in \mathcal{I}} K_{\alpha}
$$

for each $k \in\{1, \ldots, n\}$.

Since $J \backslash P_{\Omega_{1}} \neq \emptyset$ for all $P \in \mathcal{P}$, it follows that

$$
\left\{\alpha \in \mathcal{I} \mid s_{2}(\alpha) \neq \overline{0}\right\}=\cup_{k=1}^{n}\left\{\alpha \in \mathcal{I} \mid\left(s_{2} \varphi\left(j_{k}\right)\right)(\alpha) \neq \overline{0}\right\} .
$$

But $s_{2} \varphi\left(j_{k}\right) \in \bigoplus_{\alpha \in \mathcal{I}} K_{\alpha}$ for all $k \in\{1, \ldots, n\}$, and therefore $\{\alpha \in \mathcal{I} \mid$ $\left.s_{2}(\alpha) \neq \overline{0}\right\}$ is a finite union of finite sets. Thus, $\left\{\alpha \in \mathcal{I} \mid s_{2}(\alpha) \neq \overline{0}\right\}$ is finite. Hence, $s_{2} \in \bigoplus_{\alpha \in \mathcal{I}} K_{\alpha}$.

By the above arguments, it follows that $s$ and $s_{1}+s_{2}$ are elements of $\prod_{\alpha \in \mathcal{I}} K_{\alpha}=Q\left(R_{1}\right)$ which agree on the dense set $\varphi(J)$. Thus, $s=s_{1}+s_{2}$. But the above arguments also show that $s_{1}+s_{2} \in$ $\varphi\left(\left(D_{1}\right)_{\Omega_{1}}\right)+\bigoplus_{\alpha \in \mathcal{I}} K_{\alpha}=R_{1}$. Hence, $s \in R_{1}$, and it follows that $Q_{0}\left(R_{1}\right) \subseteq R_{1}$. The reverse inclusion is clear, and therefore $Q_{0}\left(R_{1}\right)=$ $R_{1}$. 
Example 4.8. Suppose that $\mathcal{P}$ is the set of all principal prime ideals of $D_{1}$. Then $R_{2}$ is a total quotient ring which satisfies $R_{2} \subsetneq Q_{0}\left(R_{2}\right) \subsetneq$ $Q\left(R_{2}\right)$ and Relation 4.1(1).

Proof. The discussion prior to Proposition 4.3 shows that $R_{2}$ is a total quotient ring. The proper inclusions will follow immediately upon establishing the validity of Relation 4.1 (1). Note that $Q_{0}\left(R_{2}\right)=R_{1}$ by Proposition 4.7. That $\Gamma\left(R_{2}\right) \not ॅ \Gamma\left(Q_{0}\left(R_{2}\right)\right)$ follows from Theorem 3.1, Proposition 4.3 and Proposition 4.4. Also, [1, Theorem 3.5] shows that a reduced total quotient ring is von Neumann regular if and only if its zero-divisor graph is complemented. In particular, $\Gamma\left(Q\left(R_{2}\right)\right)$ is complemented. On the other hand, $R_{2}$ is a total quotient ring which is not von Neumann regular (e.g., the prime ideal $\varphi(\{0\})+\bigoplus_{\alpha \in \mathcal{I}} K_{\alpha}$ is not maximal), and hence $\Gamma\left(R_{2}\right)$ is not complemented. Thus, $\Gamma\left(R_{2}\right) \nsucceq$ $\Gamma\left(Q\left(R_{2}\right)\right)$. Similarly, $\Gamma\left(Q_{0}\left(R_{2}\right)\right) \not \Gamma\left(Q\left(R_{2}\right)\right)$.

Example 4.9. Let $\mathcal{P}$ be the family of principal prime ideals belonging to the set $\left\{f D_{1} \mid f \in D_{2}\right\}$. Then $R_{2}$ is a total quotient ring which satisfies $R_{2} \subsetneq Q_{0}\left(R_{2}\right) \subsetneq Q\left(R_{2}\right)$ and Relation 4.1 (2).

Proof. The discussion prior to Proposition 4.3 shows that $R_{2}$ is a total quotient ring. The containment $R_{2} \subsetneq Q_{0}\left(R_{2}\right)$ holds since Proposition 4.7 shows that $\varphi(Z) \in Q_{0}\left(R_{2}\right) \backslash R_{2}$. That $\Gamma\left(Q_{0}\left(R_{2}\right)\right) \not$ $\Gamma\left(Q\left(R_{2}\right)\right)$ follows as in Example 4.8. This also verifies that $Q_{0}\left(R_{2}\right) \subsetneq$ $Q\left(R_{2}\right)$. Note that $\Gamma\left(R_{2}\right)$ is c.v.- $\aleph_{0}$-complete by Theorem 3.1 and Proposition 4.3. By Corollary 3.13, it only remains to show that $\left|[r]_{R_{2}}\right|=\left|[r]_{Q_{0}\left(R_{2}\right)}\right|$ for all $r \in Z\left(R_{2}\right) \backslash\{0\}$.

Let $r \in Z\left(R_{2}\right) \backslash\{0\}$. Observe that $|F| \leq\left|[r]_{R_{2}}\right|$ since $\varphi(u) r \in[r]_{R_{2}}$ for all $u \in F$. Also, the inequality $\left|[r]_{R_{2}}\right| \leq\left|[r]_{Q_{0}\left(R_{2}\right)}\right|$ follows from Lemma 2.1. Furthermore, $\mathcal{P}$ consists entirely of principal ideals, and hence $|\mathcal{I}| \leq\left|D_{1}\right|=|F|$. Since $Q_{0}\left(R_{2}\right)=R_{1}$, it follows that $\left|Q_{0}\left(R_{2}\right)\right|=|F|$. Therefore,

$$
|F| \leq\left|[r]_{R_{2}}\right| \leq\left|[r]_{Q_{0}\left(R_{2}\right)}\right| \leq\left|Q_{0}\left(R_{2}\right)\right|=|F| .
$$

Thus, $\left|[r]_{R_{2}}\right|=\left|[r]_{Q_{0}\left(R_{2}\right)}\right|$ for all $r \in Z\left(R_{2}\right) \backslash\{0\}$.

Let $R$ be a commutative $\operatorname{ring}$ and $M$ an (unitary) $R$-module. The idealization $R(+) M$ of $M$ is the commutative ring (with unity) $R \times M$, where addition is defined componentwise and multiplication is defined 
by the rule $\left(r_{1}, m_{1}\right)\left(r_{2}, m_{2}\right)=\left(r_{1} r_{2}, r_{1} m_{2}+r_{2} m_{1}\right)$. Note that $(1,0)$ is the multiplicative identity in $R(+) M$.

Define $S_{1}=\left(D_{1}\right)_{\Omega_{1}}(+) \bigoplus_{\alpha \in \mathcal{I}} K_{\alpha}$ and $S_{2}=\left(D_{2}\right)_{\Omega_{2}}(+) \bigoplus_{\alpha \in \mathcal{I}} K_{\alpha}$. Making the appropriate modifications to Proposition 4.7 will show that $S_{1}=Q_{0}\left(S_{1}\right)$. Alternatively, this is an immediate consequence of Lemma 4.5 taken together with [12, Theorem 11(f)]. If $\mathcal{P}$ consists entirely of principal ideals, then $Q_{0}\left(S_{2}\right)=S_{1}$. To see this, note that the set $\{(X,(\overline{0})),(Y,(\overline{0}))\}$ is dense and contained in $s^{-1} S_{2}$ for all $s \in S_{1}$. Therefore,

$$
S_{1} \subseteq Q_{0}\left(S_{2}\right) \subseteq Q_{0}\left(S_{1}\right)=S_{1} .
$$

Hence $Q_{0}\left(S_{2}\right)=S_{1}$.

If $(r, m) \in S_{2}$, then

$$
\operatorname{ann}_{S_{2}}((r, m))=\left\{(s, n) \in S_{2} \mid r s=0 \quad \text { and } \quad\{r n, s m\} \subseteq\{(\overline{0})\}\right\},
$$

where the inclusion $\{r n, s m\} \subseteq\{(\overline{0})\}$ holds since $r s=0$ forces either $r=0$ or $s=0$. Then it is straightforward to check that the non-zerodivisors of $S_{2}$ are precisely those elements of the form $(f / g, a)$, where $f, g \in \Omega_{2}$. Any such element is a unit in $S_{2}$ with $(f / g, a)^{-1}=(g / f, b)$, where $b(\alpha)=-(g / f)^{2} a(\alpha)$ for all $\alpha \in \mathcal{I}$. Thus, $S_{2}$ is a total quotient ring.

Example 4.10. Let $\mathcal{P}$ be the set of all principal prime ideals of $D_{1}$. Then $S_{2}$ is a total quotient ring which satisfies $\Gamma\left(S_{2}\right) \not \Gamma\left(Q_{0}\left(S_{2}\right)\right)=$ $\Gamma\left(Q\left(S_{2}\right)\right)$.

Proof. The above comments show that $S_{2}$ is a total quotient ring. Let $D \subseteq S_{2}$. Suppose that there exists a $P \in \mathcal{P}$ such that $f \in P$ for all $(f, a) \in D$. Then $(0, b) \in \operatorname{ann}_{S_{2}}(D)$, where $b$ is any element which satisfies $b(\alpha)=\overline{0}$ for all $\alpha \in \mathcal{I}$ with $P_{\alpha} \neq P$. Conversely, if no such $P$ exists, then for all $(\overline{0}) \neq b \in \bigoplus_{\alpha \in \mathcal{I}} K_{\alpha}$, there exists an element $(f, a) \in D$ such that $f b \neq(\overline{0})$. It follows that a set $D \subseteq S_{2}$ is dense if and only if it has the property that, for all $P \in \mathcal{P}$, there exist elements $f \in D_{2} \backslash P$ and $a \in \bigoplus_{\alpha \in \mathcal{I}} K_{\alpha}$ such that $(f, a) \in D$. But any element of $D_{2}$ is contained in only finitely many members of $\mathcal{P}$. Therefore, $D \subseteq S_{2}$ is dense if and only if it contains a finite set $\left\{\left(f_{i}, a_{i}\right)\right\}_{i=1}^{n}$ such that, for all $P \in \mathcal{P}$, there exists a $j \in\{1, \ldots, n\}$ with 
$f_{j} \notin P$. In particular, every dense set in $S_{2}$ contains a finite dense set. Thus, $Q_{0}\left(S_{2}\right)=Q\left(S_{2}\right)$, and hence $\Gamma\left(Q_{0}\left(S_{2}\right)\right)=\Gamma\left(Q\left(S_{2}\right)\right)$.

Note that $\Gamma\left(Q_{0}\left(S_{2}\right)\right)$ is w.c.v.- $\aleph_{0}$-complete. To see this, let $\{(f, a)$, $(g, b)\} \subseteq Q_{0}\left(S_{2}\right)$. If either $f \neq 0$ or $g \neq 0$, then let $h$ be a greatest common divisor of $f$ and $g$ in $D_{1}$. If $f=g=0$, then let $h=0$. Suppose that $c \in \bigoplus_{\alpha \in \mathcal{I}} K_{\alpha}$ is the element defined by

$$
c(\alpha)= \begin{cases}\overline{0}, & a(\alpha)=b(\alpha)=\overline{0} \\ \overline{1}, & \text { otherwise }\end{cases}
$$

Since $\mathcal{P}$ is a set of principal ideals, it follows that $\{f, g\} \subseteq P$ if and only if $h \in P(P \in \mathcal{P})$. Using this fact, it is straightforward to check that

$$
\operatorname{ann}_{Q_{0}\left(S_{2}\right)}((h, c))=\operatorname{ann}_{Q_{0}\left(S_{2}\right)}((f, a),(g, b)) .
$$

It follows that $Q_{0}\left(S_{2}\right)$ satisfies $\aleph_{0}$-(g.a.c.). Hence, $\Gamma\left(Q_{0}\left(S_{2}\right)\right)$ is w.c.v.$\aleph_{0}$-complete by the comments prior to Theorem 3.14 .

It remains to show that $\Gamma\left(S_{2}\right)$ is not w.c.v.- $\aleph_{0}$-complete. Consider the set $A=\{(X Z,(\overline{0})),(Y Z,(\overline{0}))\} \subseteq V\left(\Gamma\left(S_{2}\right)\right)$. Note that

$$
\operatorname{ann}_{S_{2}}(A)=\left\{(0, a) \in S_{2} \mid a(\alpha)=\overline{0} \text { whenever } P_{\alpha} \neq Z D_{1}\right\} .
$$

Therefore, if

$$
C(A) \backslash\{(f, b)\}=C((f, b)) \backslash A
$$

for some $(f, b) \in S_{2}$, then

$$
\{P \in \mathcal{P} \mid f \in P\}=\left\{Z D_{1}\right\} .
$$

But then $f=u Z^{n}$ for some $u \in F$ and $n \geq 1$. This contradicts that $f \in D_{2}$, and hence no such element exists. Thus, $\Gamma\left(S_{2}\right)$ is not w.c.v.$\aleph_{0}$-complete.

Let $R$ be a von Neumann regular ring. Then $R$ does not properly contain any finitely generated dense ideals. To see this, let $\left\{r_{1}, \ldots, r_{n}\right\} \subseteq R$ be dense. For each $i \in\{1, \ldots, n\}$, there exists an $s_{i} \in R$ such that $r_{i}=r_{i}^{2} s_{i}$. Then:

$$
\left(1-r_{1} s_{1}\right) \cdots\left(1-r_{n} s_{n}\right) \in \operatorname{ann}_{R}\left(r_{1}, \ldots, r_{n}\right)=\{0\} .
$$


Thus, $1=f\left(r_{1}, \ldots, r_{n}\right) \in r_{1} R+\cdots+r_{n} R$ for some $f\left(X_{1}, \ldots, X_{n}\right) \in$ $R\left[X_{1}, \ldots, X_{n}\right]$. It follows that $Q_{0}(R)=R$ whenever $R$ is von Neumann regular.

Let $\alpha$ be an ordinal. Then $Q_{\alpha}(R \oplus S)=Q_{\alpha}(R) \oplus Q_{\alpha}(S)$ for any rings $R$ and $S$ [10, Corollary 3.4]. This property will be used freely in the following examples.

Example 4.11. Suppose that $\mathcal{P}$ is the set of all principal prime ideals of $D_{1}$. Let $R$ be any von Neumann regular ring such that $R \neq Q(R)$, the isomorphism $\Gamma(R) \simeq \Gamma(Q(R))$ holds, and $|R \backslash V(\Gamma(R))|=\mid Q(R) \backslash$ $V\left(\Gamma(Q(R)) \mid\right.$. Define $W=S_{2} \oplus R$. Then $W$ is a total quotient ring which satisfies $W \subsetneq Q_{0}(W) \subsetneq Q(W)$ and Relation 4.1 (3).

Proof. Note that there exists a ring $R$ possessing the properties given in the hypothesis (e.g., [7, Example 3.5]). As the direct sum of total quotient rings, $W$ is a total quotient ring. Also, the above comments show that $Q_{0}(W)=Q_{0}\left(S_{2}\right) \oplus Q_{0}(R)=Q_{0}\left(S_{2}\right) \oplus R \subsetneq Q\left(S_{2}\right) \oplus Q(R)=$ $Q(W)$. The proper inclusion $W \subsetneq Q_{0}(W)$ will follow upon establishing Relation 4.1 (3).

The isomorphism $\Gamma\left(Q_{0}\left(S_{2}\right) \oplus R\right) \simeq \Gamma\left(Q_{0}\left(S_{2}\right) \oplus Q(R)\right)$ follows from Lemma 3.16. Also, Example 4.10 shows that $Q_{0}\left(S_{2}\right)=Q\left(S_{2}\right)$. Thus,

$$
\begin{aligned}
\Gamma\left(Q_{0}(W)\right) & =\Gamma\left(Q_{0}\left(S_{2}\right) \oplus Q_{0}(R)\right)=\Gamma\left(Q_{0}\left(S_{2}\right) \oplus R\right) \\
& \left.\simeq \Gamma\left(Q_{0}\left(S_{2}\right) \oplus Q(R)\right)=\Gamma\left(Q\left(S_{2}\right) \oplus Q(R)\right)\right) \\
& =\Gamma(Q(W)) .
\end{aligned}
$$

Note that $B(Q(R))$ is a complete Boolean algebra by [4, Theorem 11.9]. Thus $Q(R)$ satisfies (g.a.c.) by Theorem 2.4. Since $\Gamma\left(Q_{0}(R)\right)=$ $\Gamma(R) \simeq \Gamma(Q(R))$, Theorem 3.3 implies that $Q_{0}(R)$ satisfies (g.a.c.). In particular, $Q_{0}(R)$ satisfies $\aleph_{0}$-(g.a.c.). The proof of Example 4.10 shows that $Q_{0}\left(S_{2}\right)$ satisfies $\aleph_{0}$-(g.a.c.). Therefore, $\Gamma\left(Q_{0}(W)\right)$ is w.c.v.$\aleph_{0}$-complete by Theorem 3.14. However, the proof of Example 4.10 also shows that $\Gamma\left(S_{2}\right)$ is not w.c.v.- $\aleph_{0}$-complete. Hence, Theorem 3.14 implies that $\Gamma(W)$ is not w.c.v.- $\aleph_{0}$-complete. Thus $\Gamma(W) \not\left\lceil\left(Q_{0}(W)\right)\right.$. 
Example 4.12. Suppose that $\mathcal{P}$ is the family of principal prime ideals belonging to the set $\left\{f D_{1} \mid f \in D_{2}\right\}$. Then $S_{2}$ is a total quotient ring which satisfies $\Gamma\left(S_{2}\right) \simeq \Gamma\left(Q_{0}\left(S_{2}\right)\right)=\Gamma\left(Q\left(S_{2}\right)\right)$.

Proof. The comments prior to Example 4.10 show that $S_{2}$ is a total quotient ring. The equality $\Gamma\left(Q_{0}\left(S_{2}\right)\right)=\Gamma\left(Q\left(S_{2}\right)\right)$ holds as in Example 4.10. Suppose that $\left\{\left(f_{1} / g_{1}, b_{1}\right),\left(f_{2} / g_{2}, b_{2}\right)\right\} \subseteq S_{2}\left(f_{k} \in D_{2}\right.$, $\left.g_{k} \in \Omega_{2}, b_{k} \in \bigoplus_{\alpha \in \mathcal{I}} K_{\alpha}, k=1,2\right)$. If $f_{1} / g_{1}=f_{2} / g_{2}=0$, then let $h=0$. If either $f_{1} / g_{1} \neq 0$ or $f_{2} / g_{2} \neq 0$, then there exists a (finite) set $J \subseteq D_{2}$ such that $\left\{P \in \mathcal{P} \mid\left\{f_{1}, f_{2}\right\} \subseteq P\right\}=\left\{p D_{1} \mid p \in J\right\}$. If $J=\emptyset$, then let $h=1$. If $J \neq \emptyset$, then let $h=\Pi_{p \in J} p \in D_{2}$. Clearly $\left\{f_{1}, f_{2}\right\} \subseteq P$ if and only if $h \in P(P \in \mathcal{P})$. Thus $S_{2}$ satisfies $\aleph_{0}$-(g.a.c.) by the same argument used for the ring $Q_{0}\left(S_{2}\right)$ in Example 4.10. Also, $\left\{t \in S_{2} \mid t^{2}=0\right\}=\left\{(0, a) \mid a \in \bigoplus_{\alpha \in \mathcal{I}} K_{\alpha}\right\}=\left\{f \in Q_{0}\left(S_{2}\right) \mid f^{2}=0\right\}$. An argument similar to the one given in Example 4.9 shows that:

$$
|F| \leq\left|V_{t}\left(S_{2}\right)\right| \leq\left|V_{t}\left(Q_{0}\left(S_{2}\right)\right)\right| \leq\left|Q_{0}\left(S_{2}\right)\right|=|F|
$$

for all $t=(f / g, a) \in Z\left(S_{2}\right)$ with $f / g \neq 0$. Therefore, Corollary 3.11 implies that $\Gamma\left(S_{2}\right) \simeq \Gamma\left(Q_{0}\left(S_{2}\right)\right)$.

Example 4.13. Suppose that $\mathcal{P}$ is the family of principal prime ideals belonging to the set $\left\{f D_{1} \mid f \in D_{2}\right\}$. Let $R$ be any von Neumann regular ring such that $R \neq Q(R)$, the isomorphism $\Gamma(R) \simeq \Gamma(Q(R))$ holds, and $|R \backslash V(\Gamma(R))|=\mid Q(R) \backslash V\left(\Gamma(Q(R)) \mid\right.$. Define $W=S_{2} \oplus R$. Then $W$ is a total quotient ring which satisfies $W \subsetneq Q_{0}(W) \subsetneq Q(W)$ and Relation 4.1 (4).

Proof. There exists a ring $R$ possessing the properties given in the hypothesis (e.g., [7, Example 3.5]). As the direct sum of total quotient rings, $W$ is a total quotient ring. Observe that $(Z,(\overline{0})) \in Q_{0}\left(S_{2}\right) \backslash S_{2}$, and hence $W \subsetneq Q_{0}\left(S_{2}\right) \oplus Q_{0}(R)=Q_{0}(W)$. The inclusion $Q_{0}(W) \subsetneq$ $Q(W)$ holds as in Example 4.11. It remains to verify Relation 4.1(4).

Observe that $S_{k} \backslash V\left(\Gamma\left(S_{k}\right)\right)=\left\{(f / g, a) \in S_{k} \mid f, g \in \Omega_{k}\right\} \cup\{(0,(\overline{0}))\}$ for each $k \in\{0,1\}$ (cf., the comments prior to Example 4.10). But $F \subseteq \Omega_{k} \subseteq D_{1}$ and $|F|=\left|D_{1}\right|$. Hence, $\left|\Omega_{1}\right|=\left|\Omega_{2}\right|$. It is now easy to check that $\left|S_{1} \backslash V\left(\Gamma\left(S_{1}\right)\right)\right|=\left|S_{2} \backslash V\left(\Gamma\left(S_{2}\right)\right)\right|$. That is, $\left|Q_{0}\left(S_{2}\right) \backslash V\left(\Gamma\left(Q_{0}\left(S_{2}\right)\right)\right)\right|=\left|S_{2} \backslash V\left(\Gamma\left(S_{2}\right)\right)\right|$. By Lemma 3.16 and 
Example 4.12, it follows that $\Gamma\left(S_{2} \oplus R\right) \simeq \Gamma\left(Q_{0}\left(S_{2}\right) \oplus R\right)$. Thus:

$$
\Gamma(W) \simeq \Gamma\left(Q_{0}\left(S_{2}\right) \oplus R\right)=\Gamma\left(Q_{0}(W)\right),
$$

where the equality holds since $Q_{0}(R)=R$. Finally, note that the isomorphism $\Gamma\left(Q_{0}(W)\right) \simeq \Gamma(Q(W))$ holds as in Example 4.11.

It has been shown that (1), (2), (3) and (4) of Relation 4.1 can be met, in fact, by total quotient rings $R$ which satisfy $R \subsetneq Q_{0}(R) \subsetneq$ $Q(R)$. However, we do not know the answer to the following question.

Question 4.14. Does there exist a ring $R$ which satisfies Relation $4.1(5)$ ?

The remaining two examples show that an $\aleph_{\alpha}$-rationally complete ring may have a zero-divisor graph whose vertices do not satisfy any of the completeness criteria introduced in this paper. Using the fact that finite rings are rationally complete (indeed, finite rings do not properly contain any dense ideals), the comments prior to Corollary 3.15 show that it is easy to construct a rationally complete ring whose zerodivisor graph is not w.c.v. $\aleph_{0}$-complete. A less trivial example is provided in Example 4.15. Every reduced rationally complete ring has a c.v.-complete zero-divisor graph (cf., the comments prior to Corollary 3.11). However, Example 4.16 shows that a reduced $\aleph_{\alpha^{-}}$ rationally complete ring need not have a w.c.v. $\aleph_{\alpha}$-complete zerodivisor graph. In particular, the zero-divisor graph of such a ring need not be c.v. $\aleph_{\alpha}$-complete. Since a graph $\Gamma$ is c.v. $-\aleph_{\alpha}$-complete if and only if $\Gamma^{*}$ is c.v.- $\aleph_{\alpha}$-complete, the converse to Corollary 3.12 is false. Moreover, Example 4.15 shows that the conclusion of Corollary 3.15 can hold without the w.c.v.- $\aleph_{\alpha}$-complete hypothesis.

Example 4.15. Let $\mathcal{P}^{\prime}$ be the set of all principal prime ideals of $D_{1}$, and let $\mathcal{P}=\mathcal{P}^{\prime} \cup\left\{Y D_{1}+Z D_{1}\right\}$. Then $S_{1}=Q\left(S_{1}\right)$, but $\Gamma\left(S_{1}\right)$ is not w.c.v.- $\aleph_{0}$-complete. In particular, $Q\left(S_{1}\right)$ does not satisfy $\aleph_{0}$-(g.a.c.).

Proof. The equality $S_{1}=Q_{0}\left(S_{1}\right)$ holds by Lemma 4.5 together with [12, Theorem 11(f)], and $Q_{0}\left(S_{1}\right)=Q\left(S_{1}\right)$ holds as in Example 4.10. 
Note that $X D_{1}$ is the only principal prime ideal containing the set $\{X Y, X Z\}$. Therefore, if $f \in D_{1}$ and $a \in \bigoplus_{\alpha \in \mathcal{I}} K_{\alpha}$ such that

$$
\operatorname{ann}_{S_{1}}((f, a))=\operatorname{ann}_{S_{1}}((X Y,(\overline{0})),(X Z,(\overline{0}))),
$$

then $f=u X^{n}$ for some $u \in F$ and $n \geq 1$. But then $f \notin Y D_{1}+Z D_{1}$, a contradiction. Thus, no such $f$ exists. This proves the "in particular" statement. Since $D_{1}$ is an integral domain, it immediately follows that $\Gamma\left(S_{1}\right)$ is not w.c.v.- $\aleph_{0}$-complete.

Example 4.16. Let $\mathcal{P}^{\prime}$ be the set of all principal prime ideals of $D_{1}$, and let $\mathcal{P}=\mathcal{P}^{\prime} \cup\left\{Y D_{1}+Z D_{1}\right\}$. Then $R_{1}=Q_{0}\left(R_{1}\right)$, but $\Gamma\left(R_{1}\right)$ is not

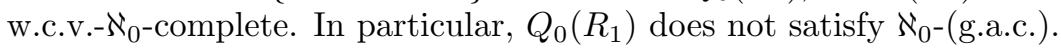

Proof. Note that $R_{1}=Q_{0}\left(R_{1}\right)$ by Proposition 4.7. Replacing $S_{1}$, $(f, a),(X Y,(\overline{0}))$ and $(X Z,(\overline{0}))$ by $R_{1}, \varphi(f)+a, \varphi(X Y)$ and $\varphi(X Z)$, respectively, the desired results follow from the proof of Example 4.15.

5. Acknowledgments. This paper embodies the finale of my Ph.D. dissertation. I would like to thank my advisor, David F. Anderson, whose suggestions toward this material have contributed to a more scholarly dissertation.

\section{REFERENCES}

1. D.F. Anderson, R. Levy and J. Shapiro, Zero-divisor graphs, von Neumann regular rings, and Boolean algebras, J. Pure Appl. Alg. 180 (2003), 221-241.

2. D.F. Anderson and P.S. Livingston, The zero-divisor graph of a commutative ring, J. Algebra 217 (1999), 434-447.

3. I. Beck, Coloring of commutative rings, J. Algebra 116 (1988), 208-226.

4. N.J. Fine, L. Gillman and J. Lambek, Rings of quotients of rings of functions, McGill University Press, Montreal, 1965.

5. A.W. Hager and J. Martinez, The ring of $\alpha$-quotients, Algebra Univ. 47 (2002), 157-182.

6. J.A. Huckaba, Commutative rings with zero divisors, Marcel-Dekker, New York, Basel, 1988.

7. J.D. LaGrange, Complemented zero-divisor graphs and Boolean rings, J. Algebra 315 (2007), 600-611.

8. ring, Int. Electron. J. Algebra 4 (2008), 63-82.

9. __ On realizing zero-divisor graphs, Comm. Alg. 36 (2008), 4509-4520. 
10. J.D. LaGrange, Rationally $\aleph_{\alpha}$-complete commutative rings of quotients, J. Algebra Appl. 8 (2009), 601-615.

11. J. Lambek, Lectures on rings and modules, Blaisdell Publishing Company, Waltham, 1966.

12. T.G. Lucas, Strong Prüfer rings and the ring of finite fractions, J. Pure Appl. Alg. 84 (1993), 59-71.

13. R. Sikorski, Boolean algebras, Springer-Verlag, New York, 1964.

Mathematics Department, University of Tennessee, Knoxville, Tn 37996 Email address: lagrangej@lindsey.edu 